\title{
Renormalization properties of a Galilean Wess-Zumino model
}

\author{
Roberto Auzzi, ${ }^{a, b}$ Stefano Baiguera, ${ }^{c}$ Giuseppe Nardelli ${ }^{a, d}$ and Silvia Penati ${ }^{c}$ \\ ${ }^{a}$ Dipartimento di Matematica e Fisica, Università Cattolica del Sacro Cuore, \\ Via Musei 41, 25121 Brescia, Italy \\ ${ }^{b}$ INFN Sezione di Perugia, \\ Via A. Pascoli, 06123 Perugia, Italy \\ ${ }^{c}$ Università degli studi di Milano Bicocca and INFN, Sezione di Milano - Bicocca, \\ Piazza della Scienza 3, 20161, Milano, Italy \\ ${ }^{d}$ TIFPA - INFN, c/o Dipartimento di Fisica, Università di Trento, \\ 38123 Povo (TN), Italy \\ E-mail: roberto.auzzi@unicatt.it, giuseppe.nardelli@unicatt.it, \\ s.baiguera@campus.unimib.it, silvia.penati@mib.infn.it
}

Abstract: We consider a Galilean $\mathcal{N}=2$ supersymmetric theory with F-term couplings in $2+1$ dimensions, obtained by null reduction of a relativistic Wess-Zumino model. We compute quantum corrections and we check that, as for the relativistic parent theory, the F-term does not receive quantum corrections. Even more, we find evidence that the causal structure of the non-relativistic dynamics together with particle number conservation constrain the theory to be one-loop exact.

Keywords: Field Theories in Lower Dimensions, Space-Time Symmetries, Superspaces, Supersymmetric Effective Theories

ARXIV EPRINT: 1904.08404 


\section{Contents}

1 Introduction 1

2 Non-relativistic supersymmetry algebra 3

2.1 Null reduction of relativistic $\mathcal{N}=1$ SUSY algebra in $3+1$ dimensions 4

2.2 Non-relativistic superspace 5

3 Review of the relativistic Wess-Zumino model $\quad 7$

3.1 Renormalization in superspace 8

3.2 Renormalization in components 9

$\begin{array}{lll}3.3 & \text { The non-renormalization theorem } & 10\end{array}$

4 The non-relativistic Wess-Zumino model 11

5 Renormalization in superspace $\quad \mathbf{1 2}$

$\begin{array}{lll}5.1 & \text { Super-Feynman and selection rules } & 13\end{array}$

$\begin{array}{lll}5.2 & \text { Renormalizability of the theory } & 15\end{array}$

$\begin{array}{lll}5.3 & \text { Loop corrections to the self-energy } & 18\end{array}$

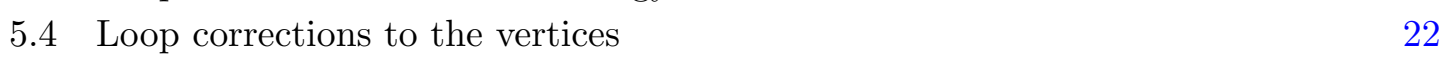

5.5 Non-relativistic non-renormalization theorem 23

6 Conclusions $\quad 24$

$\begin{array}{ll}\text { A Conventions } & 25\end{array}$

$\begin{array}{ll}\text { B Non-relativistic Wess-Zumino model in components } & 29\end{array}$

C Quantum corrections in components $\quad 30$

$\begin{array}{ll}\text { D Example of non-relativistic supergraph calculation } & 36\end{array}$

\section{Introduction}

Emergent symmetries are a recurring theme in condensed matter physics: a new symmetry may arise in the infrared, even if absent from the microscopic Hamiltonian, due to the presence of an interacting infrared fixed point in the renormalization group flow. For example, Lorentz symmetry can emerge in graphene [1-3], whose low-energy excitations can be described by massless Dirac fermions, which move with a velocity that is 300 times smaller than the speed of light.

Supersymmetry (SUSY) is a special symmetry which rotates bosonic in fermionic degrees of freedom and that has been studied for several decades, mostly from high energy 
physicist's perspective. There are several interesting settings where it appears also as an emerging symmetry in condensed matter systems. For example, superconformal invariance in two dimensions arises in the tricritical Ising model [4]. Supersymmetry also appears in the description of quantum phase transitions at the boundary of topological superconductors [5], in optical lattices [6], and in many other settings [7-11].

From a condensed-matter perspective, there are also many motivations for studying field theories with non-relativistic symmetries. This includes both systems with or without the non-relativistic boost symmetry (i.e. with Schrödinger or Lifshitz symmetry). In this work we will focus on the former, which has a richer symmetry content. Non-relativistic particles in the limit of infinite scattering length can be described by a Schrödinger conformal field theory [12-14]. This has applications in nuclear physics e.g. [15], in cold atoms [16] and in the quantum Hall effect [17]. It is then a natural question to investigate non-relativistic incarnations of SUSY, since this kind of symmetry might be emergent in the infrared of some real world systems.

The study of Galilean supersymmetry may be also interesting for holography. Almost all the examples of AdS/CFT correspondence for which the boundary theory has been precisely identified correspond to supersymmetric theories. Indeed SUSY gives a strong analytic control on several quantum physical quantities, which in some cases can be exactly computed. So, in order to find the precise holographic dual of a given gravity background which geometrically realizes the Schrödinger symmetry [18], it may be useful to focus on an explicitly supersymmetric theoretical setting.

Galilean invariance is usually thought as a low-energy approximation of theories with Poincaré invariance, and as such it can be found by performing the $c \rightarrow \infty$ limit in the corresponding relativistic setting. ${ }^{1}$ On the other hand, it is possible to obtain the Galilean group by Discrete Light Cone Quantization (DLCQ), which consists in a dimensional reduction along a null direction of a relativistic theory [20]. SUSY extensions of the Galilean algebra were first introduced in $3+1$ dimensions [21], where two super-Galilean algebras were constructed, $\mathcal{S}_{1} \mathcal{G}$ which includes a single two-component spinorial supercharge and $\mathcal{S}_{2} \mathcal{G}$, which contains two supercharges. They can be obtained as the non-relativistic limit of $\mathcal{N}=1$ and $\mathcal{N}=2$ Super-Poincaré algebras, respectively. Alternatively, $\mathcal{S}_{2} \mathcal{G}$ can be obtained performing a null reduction of the super-Poincarè algebra in $4+1$ dimensions. It turns out that $\mathcal{S}_{1} \mathcal{G} \subset \mathcal{S}_{2} \mathcal{G}$.

In $3+1$ dimensions theories with $\mathcal{S}_{1} \mathcal{G}$ and $\mathcal{S}_{2} \mathcal{G}$ invariance have been considered in [21-24], while in $2+1$ dimensions Chern-Simons theories with $\mathcal{S}_{2} \mathcal{G}$ symmetry were studied in [24-26]. Moreover, SUSY generalizations of the Schrödinger algebra have been investigated [25, 27-29], as well as Lifshitz SUSY [30].

In this paper we will build an example of a theory with $\mathcal{S}_{2} \mathcal{G}$ SUSY in $2+1$ dimensions, which we obtain by null reduction from a $3+1$ dimensional $\mathcal{N}=1$ Wess-Zumino model. A non-vanishing interaction in the superpotential which survives the null reduction requires the introduction of at least two chiral fields. Moreover, some exotic derivative cou-

\footnotetext{
${ }^{1}$ When performing this procedure, divergent expressions in the speed of light appear and we need to introduce some subtraction terms via a chemical potential and by appropriately rescaling the fields [19].
} 
plings emerge when integrating out the non-dynamical components of the fields. Similar derivative interactions were recently considered in a $1+1$ dimensional example (without supersymmetry) [31].

One may wonder if the powerful non-renormalization theorem for the $F$-term survives null-reduction, giving an interacting Galilean theory with a nice ultraviolet behaviour. We find that the non-relativistic truncation has even better UV properties:

- like the relativistic parent, the model is renormalizable and the superpotential term does not acquire quantum corrections,

- there is strong evidence that the whole renormalization of the two-point function is just at one loop (we check this claim explicitly up to four loops and discuss in general higher orders). This remarkable property is due to the $\mathrm{U}(1)$ symmetry associated to the non-relativistic particle number conservation, which limits the number of diagrams at a given perturbative order. Moreover, the causal structure strongly reduces the number of non-vanishing diagrams.

As a consequence of these two properties, we work out a set of selection rules for diagrams which simplifies the computation of the quantum corrections.

The paper is organised as follows. In section 2 we derive the $\mathcal{S}_{2} \mathcal{G}$ algebra from null reduction and we discuss the $\mathcal{N}=2$ non-relativistic superspace. In section 3 we briefly review the relativistic Wess-Zumino model and its renormalisation properties. Sections 4 and 5 contain the main results of the paper: we introduce the model and we study its quantum corrections using selection rules that we derive in the supergraph formalism. We discuss our results and possible developments in section 6. Conventions are listed in appendix A. For completeness, the model in component field formalism and its quantization are discussed in appendices B and C, respectively, while in appendix D we present in details an example of supergraph calculation.

\section{Non-relativistic supersymmetry algebra}

We are interested in studying non-relativistic SUSY theories in $2+1$ dimensions with $\mathcal{S}_{2} \mathcal{G}$ invariance. This graded generalization of the Galilean algebra contains two complex supercharges and is described by the following non-vanishing (anti)commutators

$$
\begin{aligned}
& {\left[P_{j}, K_{k}\right]=i \delta_{j k} M, \quad\left[H, K_{j}\right]=i P_{j},} \\
& {\left[P_{j}, J\right]=-i \epsilon_{j k} P_{k}, \quad\left[K_{j}, J\right]=-i \epsilon_{j k} K_{k}, \quad j, k=1,2} \\
& {[Q, J]=\frac{1}{2} Q, \quad\left\{Q, Q^{\dagger}\right\}=\sqrt{2} M,} \\
& {[\tilde{Q}, J]=-\frac{1}{2} \tilde{Q}, \quad\left[\tilde{Q}, K_{1}-i K_{2}\right]=-i Q, \quad\left\{\tilde{Q}, \tilde{Q}^{\dagger}\right\}=\sqrt{2} H,} \\
& \left\{Q, \tilde{Q}^{\dagger}\right\}=-\left(P_{1}-i P_{2}\right), \quad\left\{\tilde{Q}, Q^{\dagger}\right\}=-\left(P_{1}+i P_{2}\right)
\end{aligned}
$$

Here $P_{j}$ are the spatial components of the momentum, $K_{j}$ are the generators of Galilean boosts, $J$ is the planar angular momentum and $Q, \tilde{Q}$ are two complex supercharges. The central charge $M$ corresponds to the mass or particle number conservation. 
This is the non-relativistic $\mathcal{N}=2$ SUSY algebra in $(2+1)$ dimensions. It first appeared in the non-relativistic SUSY extension of Chern-Simons matter systems, which exhibit an enhanced superconformal symmetry [25]. Its bosonic part is the U(1) central extension of the Galilei algebra, known as Bargmann algebra. Instead, removing $\tilde{Q}$ from (2.2) we obtain the $\mathcal{S}_{1} \mathcal{G}$ algebra.

The $\mathcal{S}_{2} \mathcal{G}$ algebra can be obtained in different ways. We can start with the non-SUSY non-relativistic Galilean algebra, add two supercharges and impose consistency conditions (as done in $3+1$ dimensions [21]). Alternatively, we can perform the Inönü-Wigner contraction of the $2+1$ super-Poincarè algebra in the $c \rightarrow \infty$ limit [32]. Finally, it can be obtained by null reduction of $\mathcal{N}=1$ super-Poincaré in $3+1$ dimensions. We will follow the last approach, as it is the most convenient one for constructing the non-relativistic $\mathcal{N}=2$ superspace.

\subsection{Null reduction of relativistic $\mathcal{N}=1$ SUSY algebra in $3+1$ dimensions}

We begin by proving that the $\mathcal{S}_{2} \mathcal{G}$ algebra in (2.1), (2.2) can be obtained by null reduction of the relativistic $\mathcal{N}=1$ SUSY algebra in $3+1$ dimensions.

Given the (3+1)-dimensional Minkowski spacetime described by light-cone coordinates

$$
x^{M}=\left(x^{-}, x^{+}, x^{1}, x^{2}\right) \equiv\left(x^{-}, x^{\mu}\right) \quad x^{ \pm}=\frac{x^{3} \pm x^{0}}{\sqrt{2}}
$$

null reduction is realized by compactifying $x^{-}$on a small circle of radius $R$. For convenience, we rescale $x^{-} \rightarrow x^{-} / R$ in such a way that the rescaled coordinate is adimensional. In order to keep the metric tensor adimensional, we also rescale $x^{+} \rightarrow R x^{+}$.

It is well known that the bosonic part of the super-Poincare algebra reduces to the Bargmann algebra (2.1) by identifying some components of the linear and angular momenta with the central charge and the boost operator [18, 20].

To perform the reduction of the fermionic part of the algebra we rewrite the r.h.s. of the four-dimensional anticommutator $\left\{\mathcal{Q}_{\alpha}, \overline{\mathcal{Q}}_{\dot{\beta}}\right\}=i \sigma_{\alpha \dot{\beta}}^{M} \partial_{M}$ in terms of light-cone derivatives $\partial_{ \pm}=\frac{1}{\sqrt{2}}\left(\partial_{3} \pm \partial_{0}\right),^{2}$

$$
\{\mathcal{Q}, \overline{\mathcal{Q}}\}=i\left(\begin{array}{cc}
\sqrt{2} \partial_{+} & \partial_{1}-i \partial_{2} \\
\partial_{1}+i \partial_{2} & -\sqrt{2} \partial_{-}
\end{array}\right)
$$

When the derivatives act on local functions $\phi\left(x^{M}\right)$ of the $(3+1)$ space-time, we set $\phi\left(x^{M}\right)=e^{i m x^{-}} \varphi\left(x^{\mu}\right)$, where $m$ is an adimensional parameter. Therefore, identifying

$$
\partial_{+} \rightarrow \partial_{t}, \quad \partial_{-} \rightarrow i m
$$

and reinterpreting the four-dimensional two-spinor components as three-dimensional complex Grassmann scalars with $\mathcal{Q}_{\alpha} \rightarrow Q_{\alpha}, \overline{\mathcal{Q}}_{\dot{\beta}} \rightarrow Q_{\beta}^{\dagger}$, we obtain

$$
\begin{array}{ll}
\left\{Q_{1}, Q_{1}^{\dagger}\right\}=\sqrt{2} i \partial_{t}=\sqrt{2} H, & \left\{Q_{1}, Q_{2}^{\dagger}\right\}=i\left(\partial_{1}-i \partial_{2}\right)=-\left(P_{1}-i P_{2}\right), \\
\left\{Q_{2}, Q_{1}^{\dagger}\right\}=i\left(\partial_{1}+i \partial_{2}\right)=-\left(P_{1}+i P_{2}\right), & \left\{Q_{2}, Q_{2}^{\dagger}\right\}=-\sqrt{2} i \partial_{-}=\sqrt{2} m
\end{array}
$$

\footnotetext{
${ }^{2}$ For conventions on four-dimensional spinors see appendix A.
} 
These anticommutators coincide with the ones in (2.2) if we identify ${ }^{3}$

$$
Q_{1}=\tilde{Q}, \quad Q_{1}^{\dagger}=\tilde{Q}^{\dagger}, \quad Q_{2}=Q, \quad Q_{2}^{\dagger}=Q^{\dagger}
$$

and $m$ with the eigeinvalue of the $\mathrm{U}(1)$ generator $M$.

It is interesting to compare our result with the relativistic $\mathcal{N}=2$ SUSY algebra in $(2+1)$ dimensions that can be obtained via Kaluza-Klein reduction of the $\mathcal{N}=1$ SUSY algebra in $(3+1)$ dimensions. If we start from $\left\{\mathcal{Q}_{\alpha}, \overline{\mathcal{Q}}_{\dot{\beta}}\right\}=-\sigma_{\alpha \dot{\beta}}^{M} P_{M}$, compactify along the $x^{3}$ direction and assign momentum $p_{3} \equiv Z$, we obtain the three-dimensional anticommutator [33]

$$
\left\{\mathcal{Q}_{\alpha}, \mathcal{Q}_{\beta}^{\dagger}\right\}=-\sigma_{\alpha \beta}^{\mu} P_{\mu}+i \epsilon_{\alpha \beta} Z
$$

where $\mu=0,1,2$ and $Z$ plays the role of a central term. This expression is very similar to the one found in the non-relativistic case, eq. (2.6) with $m$ playing the role of a central charge. However, while in the relativistic reduction a central term appears in the fermionic part of the algebra when we reduce the number of dimensions, in the non-relativistic case the central charge is produced already in the bosonic sector (without requiring any SUSY extension) and accounts for the physical fact that in non-relativistic theories the particle number is a conserved quantity.

\subsection{Non-relativistic superspace}

In the relativistic setting the construction of SUSY invariant actions and the study of renormalization properties is better performed in superspace, where fields belonging to the same multiplet are organized in superfields. Having in mind to apply the same techniques to non-relativistic SUSY systems, we first construct the $\mathcal{N}=2$ non-relativistic superspace by applying null reduction to the four-dimensional one. The non-relativistic superspace was first introduced in four dimensions [22, 23], whereas previous constructions in three dimensions based on different techniques can be found in [26, 34].

We start with the relativistic $\mathcal{N}=1$ superspace in $(3+1)$ described by superspace coordinates $\left(x^{\mu}, \theta^{\alpha}, \bar{\theta}^{\dot{\alpha}}\right)$. An explicit realization of the super-Poincaré algebra is given in terms of the following supercharges ${ }^{4}$

$$
\mathcal{Q}_{\alpha}=i \frac{\partial}{\partial \theta^{\alpha}}-\frac{1}{2} \bar{\theta}^{\dot{\beta}} \partial_{\alpha \dot{\beta}}, \quad \overline{\mathcal{Q}}_{\dot{\alpha}}=-i \frac{\partial}{\partial \bar{\theta}^{\dot{\alpha}}}+\frac{1}{2} \theta^{\beta} \partial_{\beta \dot{\alpha}}
$$

and SUSY covariant derivatives

$$
\mathcal{D}_{\alpha}=\frac{\partial}{\partial \theta^{\alpha}}-\frac{i}{2} \bar{\theta}^{\dot{\beta}} \partial_{\alpha \dot{\beta}}, \quad \overline{\mathcal{D}}_{\dot{\alpha}}=\frac{\partial}{\partial \bar{\theta}^{\dot{\alpha}}}-\frac{i}{2} \theta^{\beta} \partial_{\beta \dot{\alpha}}
$$

which act on local superfields $\Psi\left(x^{M}, \theta^{\alpha}, \bar{\theta}^{\dot{\alpha}}\right)$.

\footnotetext{
${ }^{3}$ We note that identification (2.7) is required to obtain the correct anticommutators $\left\{Q, Q^{\dagger}\right\}$ and $\left\{\tilde{Q}, \tilde{Q}^{\dagger}\right\}$, but it interchanges $\left(P_{1}+i P_{2}\right)$ and $\left(P_{1}-i P_{2}\right)$ in the mixed anticommutators. This is simply due to the fact that we chose $x^{-}$as the compact light-cone coordinate. Had we chosen $x^{+}$we would have obtained exactly the algebra in $(2.2)$. Since having $\left(P_{1}+i P_{2}\right)$ and $\left(P_{1}-i P_{2}\right)$ interchanged does not affect our construction, we take $x^{-}$as the compact direction being this a more conventional choice in the literature.

${ }^{4}$ Superspace conventions are discussed in appendix A.
} 
The $\mathcal{N}=2$ non-relativistic superspace in $(2+1)$ dimensions can be easily obtained by suitably generalizing the DLCQ procedure. To this end, we move to light-cone coordinates (2.3) and rewrite $\partial_{\alpha \dot{\beta}}=\sigma_{\alpha \dot{\beta}}^{M} \partial_{M}$ in (2.9), (2.10) in terms of $\partial_{ \pm}, \partial_{1}, \partial_{2}$. Then, mimicking what we have done in the non-supersymmetric case, we reduce a generic four-dimensional field as $\phi\left(x^{M}\right)=e^{i m x^{-}} \varphi\left(x^{\mu}\right)$. Since supersymmetry requires each field component of a multiplet to be an eigenfunction of the $\partial_{-}$operator with the same eigenvalue $m$, the reduction can be done directly at the level of superfields, by writing

$$
\Psi\left(x^{M}, \theta^{\alpha}, \bar{\theta}^{\dot{\alpha}}\right)=e^{i m x^{-}} \tilde{\Psi}\left(x^{+} \equiv t, x^{i}, \theta^{\alpha},\left(\theta^{\alpha}\right)^{\dagger}\right)
$$

Acting on these superfields with supercharges and covariant derivatives (2.9), (2.10) rewritten in terms of light-cone derivatives, and performing the identification $\partial_{+} \equiv \partial_{t}$ and $\partial_{-} \equiv i M$ (with eigenvalue $m$ ), we obtain ${ }^{5}$

$$
\left\{\begin{array} { l } 
{ Q _ { 1 } = i \frac { \partial } { \partial \theta ^ { 1 } } - \frac { 1 } { 2 } \overline { \theta } ^ { 2 } ( \partial _ { 1 } - i \partial _ { 2 } ) - \frac { 1 } { \sqrt { 2 } } \overline { \theta } ^ { 1 } \partial _ { t } } \\
{ \overline { Q } _ { 1 } = - i \frac { \partial } { \partial \overline { \theta } ^ { 1 } } + \frac { 1 } { 2 } \theta ^ { 2 } ( \partial _ { 1 } + i \partial _ { 2 } ) + \frac { 1 } { \sqrt { 2 } } \theta ^ { 1 } \partial _ { t } } \\
{ Q _ { 2 } = i \frac { \partial } { \partial \theta ^ { 2 } } - \frac { 1 } { 2 } \overline { \theta } ^ { 1 } ( \partial _ { 1 } + i \partial _ { 2 } ) - \frac { i } { \sqrt { 2 } } \overline { \theta } ^ { 2 } M } \\
{ \overline { Q } _ { 2 } = - i \frac { \partial } { \partial \overline { \theta } ^ { 2 } } + \frac { 1 } { 2 } \theta ^ { 1 } ( \partial _ { 1 } - i \partial _ { 2 } ) - \frac { i } { \sqrt { 2 } } \theta ^ { 2 } M }
\end{array} \quad \left\{\begin{array}{l}
D_{1}=\frac{\partial}{\partial \theta^{1}}-\frac{i}{2} \bar{\theta}^{2}\left(\partial_{1}-i \partial_{2}\right)-\frac{i}{\sqrt{2}} \bar{\theta}^{1} \partial_{t} \\
\bar{D}_{1}=\frac{\partial}{\partial \bar{\theta}^{1}}-\frac{i}{2} \theta^{2}\left(\partial_{1}+i \partial_{2}\right)-\frac{i}{\sqrt{2}} \theta^{1} \partial_{t} \\
D_{2}=\frac{\partial}{\partial \theta^{2}}-\frac{i}{2} \bar{\theta}^{1}\left(\partial_{1}+i \partial_{2}\right)-\frac{1}{\sqrt{2}} \bar{\theta}^{2} M \\
\bar{D}_{2}=\frac{\partial}{\partial \bar{\theta}^{2}}-\frac{i}{2} \theta^{1}\left(\partial_{1}-i \partial_{2}\right)-\frac{1}{\sqrt{2}} \theta^{2} M
\end{array}\right.\right.
$$

These operators realize a representation of the non-relativistic algebra (2.2) and can be interpreted as the supercharges and the covariant derivatives of a three-dimensional $\mathcal{N}=2$ superspace described by coordinates $\left(t, x^{1}, x^{2}, \theta^{1}, \theta^{2}, \bar{\theta}^{1}, \bar{\theta}^{2}\right)$. Correspondingly, the functions $\tilde{\Psi}$ in (2.11) are three-dimensional $\mathcal{N}=2$ superfields realizing a representation of the nonrelativistic SUSY algebra.

We point out that the non-relativistic superspace and the corresponding supercharges could be alternatively constructed by quotienting the SUSY extension of the Bargmann algebra by the subgroup of spatial rotations and Galilean boosts, in analogy with the construction of the relativistic superspace as the quotient super-Poincaré/SO(1,3). However, the null reduction procedure is more convenient, as it relies on the quotient algebra already implemented in four dimensions.

As in the relativistic case, imposing suitable constraints we can reduce the number of superfield components and realize irreducible representations of the superalgebra. In particular, we are interested in the construction of (anti)chiral superfields. These can be obtained either by null reduction of the four-dimensional (anti)chiral superfields, $\overline{\mathcal{D}}_{\dot{\alpha}} \Sigma=0$ $\left(\mathcal{D}_{\alpha} \bar{\Sigma}=0\right)$, or directly in three-dimensional superspace by imposing

$$
\bar{D}_{\alpha} \Sigma=0, \quad D_{\alpha} \bar{\Sigma}=0
$$

where the covariant derivatives are given in (2.12).

Defining coordinates

$$
x_{L, R}^{\mu}=x^{\mu} \mp i \theta^{\alpha}\left(\bar{\sigma}^{\mu}\right)_{\alpha \beta} \bar{\theta}^{\beta} \quad \mu=+, 1,2
$$

\footnotetext{
${ }^{5}$ From now on we rename $\left(\theta^{\alpha}\right)^{\dagger} \equiv \bar{\theta}^{\alpha}$ and similarly for the other grassmannian quantities.
} 
which satisfy $\bar{D}_{\alpha} x_{L}^{\mu}=0, D_{\alpha} x_{R}^{\mu}=0$, the (anti)chiral superfields have the following expansion

$$
\begin{aligned}
& \Sigma\left(x_{L}^{\mu}, \theta^{\alpha}\right)=\varphi\left(x_{L}^{\mu}\right)+\theta^{\alpha} \tilde{\psi}_{\alpha}\left(x_{L}^{\mu}\right)-\theta^{2} F\left(x_{L}^{\mu}\right) \\
& \bar{\Sigma}\left(x_{R}^{\mu}, \bar{\theta}^{\beta}\right)=\bar{\varphi}\left(x_{R}^{\mu}\right)+\bar{\theta}_{\gamma} \overline{\tilde{\psi}}^{\gamma}\left(x_{R}^{\mu}\right)-\bar{\theta}^{2} \bar{F}\left(x_{R}^{\mu}\right)
\end{aligned}
$$

Manifestly supersymmetric actions can be constructed by using the Berezin integral on spinorial coordinates. In the relativistic superspace, for a generic superfield $\Psi$ we define

$$
\int d^{4} x d^{4} \theta \Psi=\left.\int d^{4} x \mathcal{D}^{2} \overline{\mathcal{D}}^{2} \Psi\right|_{\theta=\bar{\theta}=0}
$$

with covariant derivatives given in (2.10). Performing the null reduction and extracting the $x^{-}$dependence of the superfield by setting $\Psi=e^{i m x^{-}} \tilde{\Psi}$, we obtain the prescription for the Berezin integrals in the non-relativistic superspace

$$
\begin{aligned}
\int d^{4} x d^{4} \theta \Psi & =\left.\int d^{4} x \mathcal{D}^{2} \overline{\mathcal{D}}^{2} \Psi\right|_{\theta=\bar{\theta}=0} \longrightarrow \\
\left.\int d^{3} x D^{2} \bar{D}^{2} \tilde{\Psi}\right|_{\theta=\bar{\theta}=0} \times \frac{1}{2 \pi} \int_{0}^{2 \pi} d x^{-} e^{i m x^{-}} & \equiv \int d^{3} x d^{4} \theta \tilde{\Psi} \times \frac{1}{2 \pi} \int_{0}^{2 \pi} d x^{-} e^{i m x^{-}}
\end{aligned}
$$

where in the r.h.s. $d^{3} x \equiv d t d x^{1} d x^{2}$ and the spinorial derivatives are the ones in eq. (2.12). It is immediate to observe that whenever $m \neq 0$ we obtain a trivial reduction due to the $x^{-}$ integral. Non-vanishing expressions arise only if the super-integrand $\Psi$ is uncharged respect to the mass generator. In the construction of SUSY invariant actions this is equivalent to require the action to be invariant under one extra global $\mathrm{U}(1)$ symmetry [23].

\section{Review of the relativistic Wess-Zumino model}

In this section we briefly review the renormalization of the relativistic four-dimensional Wess-Zumino (WZ) model, both in superspace and in components, in order to fix our notations and recall the main physical properties that we plan to investigate in a nonrelativistic set-up.

The classical action of the WZ model [35] in $(3+1)$ dimensions is given by

$$
S=\int d^{4} x d^{4} \theta \bar{\Sigma} \Sigma+\int d^{4} x d^{2} \theta\left(\frac{m}{2} \Sigma^{2}+\frac{\lambda}{3 !} \Sigma^{3}\right)+\text { h.c. }
$$

and describes the dynamics of the field components of a chiral superfield $\Sigma=(\phi, \psi, F)$. For simplicity we focus on the massless model, so from now on we set $m=0$. This model is classically scale invariant.

When reduced in components using definitions (A.18) the action reads

$$
S=\int d^{4} x\left[-\partial^{M} \bar{\phi} \partial_{M} \phi+i \bar{\psi} \bar{\sigma}^{M} \partial_{M} \psi+\bar{F} F+\left(3 \lambda F \phi^{2}-3 \lambda \psi^{\alpha} \psi_{\alpha} \phi+\text { h.c. }\right)\right]
$$

The action in (3.1) is manifestly invariant under $\mathcal{N}=1$ SUSY transformations

$$
\delta_{\varepsilon} \Sigma=\left[i \varepsilon^{\alpha} \mathcal{Q}_{\alpha}+i \bar{\varepsilon}_{\dot{\alpha}} \overline{\mathcal{Q}}^{\dot{\alpha}}, \Sigma\right]
$$


Equivalently, action (3.2) is invariant under

$$
\left\{\begin{array}{l}
\delta_{\varepsilon} \phi=-\varepsilon^{\alpha} \psi_{\alpha} \\
\delta_{\varepsilon} \psi_{\alpha}=i \bar{\varepsilon}^{\dot{\alpha}}\left(\partial_{\alpha \dot{\alpha}} \phi\right)+\varepsilon_{\alpha} F \\
\delta_{\varepsilon} F=-i \bar{\varepsilon}^{\dot{\alpha}} \partial_{\alpha \dot{\alpha}} \psi^{\alpha}
\end{array}\right.
$$

\subsection{Renormalization in superspace}

At quantum level we consider the generating functional

$$
Z[J, \bar{J}]=\int \mathcal{D} \Sigma \mathcal{D} \bar{\Sigma} \exp \left\{i\left(S+\int d^{2} \theta J \Sigma+\int d^{2} \bar{\theta} \bar{J} \bar{\Sigma}\right)\right\}
$$

where the sources $J, \bar{J}$ are chiral and anti-chiral superfields, respectively. Correlation functions can be obtained by repeated application of functional derivatives

$$
\frac{\delta J\left(z_{i}\right)}{\delta J\left(z_{j}\right)}=\overline{\mathcal{D}}^{2} \delta^{(8)}\left(z_{i}-z_{j}\right), \quad \frac{\delta \bar{J}\left(z_{i}\right)}{\delta \bar{J}\left(z_{j}\right)}=\mathcal{D}^{2} \delta^{(8)}\left(z_{i}-z_{j}\right)
$$

where $z \equiv\left(x^{M}, \theta^{\alpha}, \bar{\theta}^{\dot{\alpha}}\right)$ and $\delta^{(8)}\left(z_{i}-z_{j}\right) \equiv \delta^{(4)}\left(x_{i}-x_{j}\right) \delta^{(2)}\left(\theta_{i}-\theta_{j}\right) \delta^{(2)}\left(\bar{\theta}_{i}-\bar{\theta}_{j}\right)$. The additional covariant derivatives acting on the delta functions come from the fact that we are deriving constrained superfields.

Renormalizability properties can be investigated in superspace, where Feynman rules can be formulated directly for superfields. These allow to draw supergraphs which can be eventually reduced to ordinary Feynman integrals by performing D-algebra.

In short, for the massless WZ model super-Feynman rules are [36]

- Superfield propagator

$$
\left\langle\Sigma\left(z_{i}\right) \bar{\Sigma}\left(z_{j}\right)\right\rangle=\frac{1}{\square} \delta^{(8)}\left(z_{i}-z_{j}\right) \longrightarrow\langle\Sigma(p) \bar{\Sigma}(-p)\rangle=-\frac{1}{p^{2}} \delta^{(4)}\left(\theta_{i}-\theta_{j}\right)
$$

- Vertices. These are read directly from the interaction Lagrangian. They are cubic vertices containing only chiral or anti-chiral superfields. Because of identity (3.6) we assign one $\overline{\mathcal{D}}^{2}\left(\mathcal{D}^{2}\right)$ to every internal line exiting from a chiral (anti-chiral) vertex. One of these factors is then used to complete the chiral (anti-chiral) integral at the vertex, thus dealing only with $\int d^{4} \theta$ at each vertex.

At this point we need to perform the spinorial integrals exploiting the spinorial delta functions, in order to obtain a final result which is a local function of $(\theta, \bar{\theta})$ integrated in $d^{4} \theta$. However, we need to take into account that spinorial deltas may be partially affected by residual $\mathcal{D}$ 's or $\overline{\mathcal{D}}$ 's acting on internal lines. Moreover, products of identical deltas are subject to (we set $\delta_{i j} \equiv \delta^{(2)}\left(\theta_{i}-\theta_{j}\right) \delta^{(2)}\left(\bar{\theta}_{i}-\bar{\theta}_{j}\right)$ )

$$
\begin{aligned}
& \delta_{i j} \delta_{i j}=0, \quad \delta_{i j} \mathcal{D}^{\alpha} \delta_{i j}=0, \quad \delta_{i j} \mathcal{D}^{2} \delta_{i j}=0, \quad \delta_{i j} \mathcal{D}^{\alpha} \overline{\mathcal{D}}^{\dot{\alpha}} \delta_{i j}=0, \quad \delta_{i j} \mathcal{D}^{\alpha} \overline{\mathcal{D}}^{2} \delta_{i j}=0 \\
& \delta_{i j} \mathcal{D}^{\alpha} \overline{\mathcal{D}}^{2} \mathcal{D}^{\beta} \delta_{i j}=-\epsilon^{\alpha \beta} \delta_{i j}, \quad \delta_{i j} \mathcal{D}^{2} \overline{\mathcal{D}}^{2} \delta_{i j}=\delta_{i j} \overline{\mathcal{D}}^{2} \mathcal{D}^{2} \delta_{i j}=\delta_{i j} \frac{\mathcal{D}^{\alpha} \overline{\mathcal{D}}^{2} \mathcal{D}_{\alpha}}{2} \delta_{i j}=\delta_{i j}
\end{aligned}
$$


Therefore, it is easy to verify that we need to perform D-algebra until we reach a configuration in which exactly two $\mathcal{D}$ 's and two $\overline{\mathcal{D}}$ 's survive in each loop. This amounts to integrate by parts spinorial derivatives at the vertices and trade products of them with space-time derivatives through commutation rules like

$$
\left[\mathcal{D}^{\alpha}, \overline{\mathcal{D}}^{2}\right]=i \partial^{\alpha \dot{\alpha}} \overline{\mathcal{D}}_{\dot{\alpha}}, \quad\left[\overline{\mathcal{D}}^{\dot{\alpha}}, \mathcal{D}^{2}\right]=-i \partial^{\alpha \dot{\alpha}} \mathcal{D}_{\alpha}, \quad \mathcal{D}^{2} \overline{\mathcal{D}}^{2} \mathcal{D}^{2}=\square \mathcal{D}^{2}, \quad \overline{\mathcal{D}}^{2} \mathcal{D}^{2} \overline{\mathcal{D}}^{2}=\square \overline{\mathcal{D}}^{2}
$$

Whenever in a loop we end up with a number of derivatives which less than $2 \mathcal{D}$ 's $+2 \overline{\mathcal{D}}$ 's the configuration vanishes and can be discharged. Instead, when in a loop we are left with two $\mathcal{D}$ 's plus two $\overline{\mathcal{D}}$ 's the spinorial integrations associated to that loop can be performed and we are left with a non-vanishing expression local in the spinorial coordinates.

In so doing, we reduce a supergraph to the sum of a number of ordinary Feynman diagrams. As usual, in momentum space these correspond to integrals over loop momenta, with momentum conserved at each vertex. In general UV and IR divergences arise, which require suitable regularizations to perform the integrals. At the end of the calculation, going back to configuration space we obtain contributions that are given by local functions of the superspace coordinates integrated in $d^{4} x d^{4} \theta$.

The WZ model is renormalizable by power counting. Applying the supergraph techniques described above, it immediately follows that UV divergences always arise in the form of non-chiral superspace integrals and, as such, can only contribute to the kinetic part of the effective action. The cubic superpotential, at the contrary, never gets divergent corrections, and consequently it does not undergo any renormalization. This is the proof of the well-known perturbative non-renormalization theorem [37].

Cancellation of loop divergences requires a wavefunction renormalization. Due to the non-renormalization theorem, the coupling constant of the model inherits a non-trivial renormalization as well. In fact,

$$
\mathcal{L}=\int d^{4} \theta(\bar{\Sigma} \Sigma)+\int d^{2} \theta\left(\lambda \Sigma^{3}\right) \rightarrow \mathcal{L}_{\text {ren }}=\int d^{4} \theta Z_{\Sigma}(\bar{\Sigma} \Sigma)+\int d^{2} \theta Z_{\lambda} Z_{\Sigma}^{3 / 2}\left(\lambda \Sigma^{3}\right)
$$

but the absence of chiral divergences implies

$$
Z_{\lambda} Z_{\Sigma}^{3 / 2}=1 \Longrightarrow Z_{\lambda}=Z_{\Sigma}^{-3 / 2}
$$

\subsection{Renormalization in components}

It is interesting to see how the non-renormalization theorem is formulated when we perform perturbative calculations in components. In view of the comparison with the nonrelativistic analysis, this is motivated by the fact that so far most of the literature on non-relativistic systems has used the component formalism.

Starting with the action in components given in (3.2) we can easily obtain the corresponding Feynman rules. If we do not eliminate the auxiliary fields the propagators are the ordinary scalar and fermion propagators completed with $\langle F \bar{F}\rangle=1,{ }^{6}$ while the vertices are still cubic vertices, as inferred directly from the action.

\footnotetext{
${ }^{6}$ They can also be obtained by reducing the super-propagator (3.7) in components.
} 
Evaluating ordinary Feynman diagrams and isolating the UV divergent terms, the renormalizability of the model allows to write

$$
\mathcal{L}_{\text {ren }}=-Z \partial^{M} \bar{\phi} \partial_{M} \phi+i Z \bar{\psi} \bar{\sigma}^{M} \partial_{M} \psi+Z \bar{F} F+\left(3 \lambda Z_{\lambda} Z^{3 / 2} F \phi^{2}+3 \lambda Z_{\lambda} Z^{3 / 2} \psi^{\alpha} \psi_{\alpha} \phi+\text { h.c. }\right)
$$

where we have used the SUSY condition $Z_{\phi}=Z_{\psi}=Z_{F} \equiv Z$. It follows that the nonrenormalization theorem still leads to condition (3.11). In fact, since we have not eliminated the auxiliary field $F$, this is nothing but a trivial rephrasing of the superspace approach.

Instead, we can proceed by first integrating out the auxiliary field $F$ from the action, using its equations of motion. Performing the perturbative analysis and taking into account the non-renormalization condition (3.11), it turns out that the renormalized action for the dynamical fields reads

$$
\mathcal{L}_{\text {ren }}=-Z \partial^{M} \bar{\phi} \partial_{M} \phi+i Z \bar{\psi} \bar{\sigma}^{M} \partial_{M} \psi+\left(3 \lambda \psi^{\alpha} \psi_{\alpha} \phi-9 Z^{-1}|\lambda|^{2}|\phi|^{4}+\text { h.c. }\right)
$$

The relevant fact is that while the cubic vertex is still non-renormalized, the quartic scalar interaction renormalizes non-trivially, due to the wavefunction renormalization. This shows that when working in components and integrating out the auxiliary fields, quantum corrections to the vertices may arise, although the non-renormalization theorem is still at work.

\subsection{The non-renormalization theorem}

The holomorphicity of the superpotential is a powerful constraint which forces all quantum corrections to $F$-terms to vanish. At perturbative level, a direct proof can be obtained by supergraphs technique, as reviewed above. The non-perturbative derivation of this result follows instead from an argument due to Seiberg [38].

Here we quickly review the argument, following [39]. We consider a WZ model for $n$ chiral superfields $\Sigma_{a}$ interacting through a generic superpotential $W$

$$
S=\int d^{4} x d^{4} \theta \bar{\Sigma}_{a} \Sigma_{a}+\int d^{4} x d^{2} \theta W\left(\Sigma_{a}\right)+\text { h.c. }
$$

We introduce one extra chiral superfield $Y$, whose scalar part is set to 1 to recover the original action, whereas the spinorial and auxiliary components vanish identically. We assign R-charges $R\left(\Sigma_{a}\right)=0$ and $R(Y)=2$. We also introduce real superfields $Z_{a b}$ for the wave function renormalization

$$
\tilde{S}=\int d^{4} x d^{4} \theta Z_{a b} \bar{\Sigma}_{a} \Sigma_{b}+\int d^{4} x d^{2} \theta Y W\left(\Sigma_{a}\right)+\text { h.c. }
$$

Assuming that the regularization procedure does not spoil SUSY, the Wilsonian effective action at a given scale $\lambda$ is of the following form

$$
\tilde{S}_{\lambda}=\int d^{4} x d^{4} \theta K\left(\bar{\Sigma}_{a} \Sigma_{a}, Z_{a b}, Y, \bar{Y}, \mathcal{D}\right)+\int d^{4} x d^{2} \theta W_{\lambda}\left(\Sigma_{a}, Y\right)+\text { h.c. }
$$

Then R-invariance and holomorphicity of the superpotential force $W_{\lambda}$ to be of the form

$$
W_{\lambda}\left(\Sigma_{a}, Y\right)=Y W_{\lambda}\left(\Sigma_{a}\right)
$$

Taking the weak coupling limit $Y \rightarrow 0$, the only contribution to the superpotential is a tree-level vertex, and therefore we find $W_{\lambda}\left(\Sigma_{a}\right)=W\left(\Sigma_{a}\right)$. 


\section{The non-relativistic Wess-Zumino model}

We now study the non-relativistic counterpart of the WZ model using the superfield formulation of section 2.2. We are primarily interested in investigating if and how the renormalization properties of the relativistic model survive in this case.

The natural way to obtain the non-relativistic version of the WZ model is by applying null reduction (2.18) to the action in (3.1). Setting $\Sigma=e^{i m x^{-}} \Phi$ there, we immediately see that while the canonical Kahler potential survives the reduction being $U(1)$ neutral, the holomorphic superpotential has charge 3 and is killed by the $x^{-}$integration. The only way-out to obtain an interacting non-relativistic scalar model is then to introduce at least two species of superfields with different $m$ charges, and trigger them in such a way that also the superpotential turns out to be neutral.

We then start in four dimensions with a WZ model for two massless fields described by the action

$$
S=\int d^{4} x d^{4} \theta\left(\bar{\Sigma}_{1} \Sigma_{1}+\bar{\Sigma}_{2} \Sigma_{2}\right)+g \int d^{4} x d^{2} \theta \Sigma_{1}^{2} \Sigma_{2}+\text { h.c. }
$$

We perform the null reduction by setting

$$
\Sigma_{1}\left(x^{M}, \theta, \bar{\theta}\right)=\Phi_{1}\left(x^{\mu}, \theta, \bar{\theta}\right) e^{i m x^{-}}, \quad \Sigma_{2}\left(x^{M}, \theta, \bar{\theta}\right)=\Phi_{2}\left(x^{\mu}, \theta, \bar{\theta}\right) e^{-2 i m x^{-}}
$$

so that the superpotential is neutral under the mass generator. The reduced action reads

$$
S=\int d^{3} x d^{4} \theta\left(\bar{\Phi}_{1} \Phi_{1}+\bar{\Phi}_{2} \Phi_{2}\right)+g \int d^{3} x d^{2} \theta \Phi_{1}^{2} \Phi_{2}+\text { h.c. }
$$

We will refer to the superfields in eq. (4.2) as belonging to sector 1 and 2, respectively. Since in the non-relativistic superspace the time coordinate has twice the dimensions of the spatial ones, superfields have still mass dimension one and the coupling $g$ is dimensionless. Therefore the model shares classical scale invariance with its relativistic counterpart.

This action is invariant under the non-relativistic $\mathcal{N}=2$ supersymmetry. Using definition (2.18) for the non-relativistic spinorial integrals, we can reduce it to components.

Focusing first on the kinetic part of the action, we can integrate out the auxiliary fields and obtain (for details see appendix B)

$$
\begin{aligned}
S_{\text {kin }}= & \int d^{3} x\left[2 i m \bar{\varphi}_{1} \partial_{t} \varphi_{1}+\bar{\varphi}_{1} \partial_{i}^{2} \varphi_{1}-4 i m \bar{\varphi}_{2} \partial_{t} \varphi_{2}+\bar{\varphi}_{2} \partial_{i}^{2} \varphi_{2}\right. \\
& \left.+2 i m \bar{\chi}_{1} \partial_{t} \chi_{1}+\bar{\chi}_{1} \partial_{i}^{2} \chi_{1}+4 i m \bar{\chi}_{2} \partial_{t} \chi_{2}-\bar{\chi}_{2} \partial_{i}^{2} \chi_{2}\right]
\end{aligned}
$$

where $\varphi_{1,2}$ and $\chi_{1,2}$ are the dynamical non-relativistic scalar and fermion fields, respectively.

If we apply Fourier transform

$$
\varphi\left(x^{\mu}\right)=\int \frac{d \omega d^{2} k}{(2 \pi)^{3}} a(\vec{k}) e^{-i(\omega t-\vec{k} \cdot \vec{x})}
$$

to both scalars and fermions, the free equations of motion lead to the following dispersion relations

$$
\omega_{1}=\frac{{\overrightarrow{k_{1}}}^{2}}{2 m} \quad \omega_{2}=-\frac{{\overrightarrow{k_{2}}}^{2}}{4 m}
$$


The wrong sign for the energy of $\varphi_{2}$ and $\chi_{2}$ is due to $U(1)$ invariance which forces to assign a negative eigenvalue to the mass operator for $\Phi_{2}$ in decomposition (4.2).

To circumvent this problem we first integrate by parts $\partial_{t}, \partial_{i}^{2}$ in sector 2 , obtaining

$$
\begin{aligned}
S_{\text {kin }}= & \int d^{3} x\left[2 i m \bar{\varphi}_{1} \partial_{t} \varphi_{1}+\bar{\varphi}_{1} \partial_{i}^{2} \varphi_{1}+4 i m \varphi_{2} \partial_{t} \bar{\varphi}_{2}+\varphi_{2} \partial_{i}^{2} \bar{\varphi}_{2}\right. \\
& \left.+2 i m \bar{\chi}_{1} \partial_{t} \chi_{1}+\bar{\chi}_{1} \partial_{i}^{2} \chi_{1}+4 i m \chi_{2} \partial_{t} \bar{\chi}_{2}+\chi_{2} \partial_{i}^{2} \bar{\chi}_{2}\right]
\end{aligned}
$$

Then we interchange the roles of $\varphi_{2}$ and $\bar{\varphi}_{2}$ and similarly of $\chi_{2}$ and $\bar{\chi}_{2}$. This operation is equivalent to reversing the role of creation and annihilation operators. At the level of superfields this amounts to interchanging all the components of $\Phi_{2}$ with the components of $\bar{\Phi}_{2}$. Note that this operation is done without exchanging the grassmannian coordinates, i.e. without changing the chirality of the superfield. From now on we name $\Phi_{2}$ the chiral superfield whose components ${ }^{7}$ are $\left(\bar{\varphi}_{2}, \bar{\xi}_{2}, \bar{\chi}_{2}, \bar{F}_{2}\right)$, while the antichiral $\bar{\Phi}_{2}$ has components $\left(\varphi_{2}, \xi_{2}, \chi_{2}, F_{2}\right)$, and assign positive mass $2 m$ to $\Phi_{2}$.

Under this exchange and having eliminated the auxiliary fields, the complete action in components reads

$$
\begin{aligned}
S= & \int d^{3} x\left[2 i m \bar{\varphi}_{1} \partial_{t} \varphi_{1}+\bar{\varphi}_{1} \partial_{i}^{2} \varphi_{1}+4 i m \bar{\varphi}_{2} \partial_{t} \varphi_{2}+\bar{\varphi}_{2} \partial_{i}^{2} \varphi_{2}\right. \\
& +2 i m \bar{\chi}_{1} \partial_{t} \chi_{1}+\bar{\chi}_{1} \partial_{i}^{2} \chi_{1}+4 i m \bar{\chi}_{2} \partial_{t} \chi_{2}+\bar{\chi}_{2} \partial_{i}^{2} \chi_{2}-4|g|^{2}\left|\varphi_{1} \varphi_{2}\right|^{2}-|g|^{2}\left|\varphi_{1}\right|^{4} \\
& -i g\left(\sqrt{2} \varphi_{1} \chi_{1}\left(\partial_{1}-i \partial_{2}\right) \bar{\chi}_{2}-2 \bar{\varphi}_{2} \chi_{1}\left(\partial_{1}-i \partial_{2}\right) \chi_{1}+2 \sqrt{2} \varphi_{1}\left(\left(\partial_{1}-i \partial_{2}\right) \chi_{1}\right) \bar{\chi}_{2}\right)+\text { h.c. } \\
& \left.+2|g|^{2}\left(-\left|\varphi_{1}\right|^{2} \bar{\chi}_{1} \chi_{1}-4\left|\varphi_{1}\right|^{2} \bar{\chi}_{2} \chi_{2}+2\left|\varphi_{2}\right|^{2} \bar{\chi}_{1} \chi_{1}+2 \sqrt{2} \varphi_{1} \varphi_{2} \bar{\chi}_{1} \bar{\chi}_{2}+2 \sqrt{2} \bar{\varphi}_{1} \bar{\varphi}_{2} \chi_{2} \chi_{1}\right)\right]
\end{aligned}
$$

We note the presence of cubic derivative interactions, together with the standard quartic couplings. Similar derivative interactions in a non-supersymmetric $1+1$ dimensional Galilean model have been recently studied in [31].

We remark that the same action could be obtained by null reduction of the $3+1$ relativistic WZ action in components where the auxiliary fields have been integrated out. Similar computations were performed in [40].

\section{Renormalization in superspace}

We now study the renormalization properties of the model defined by eq. (4.3), using superspace formalism. ${ }^{8}$ To this purpose, we first collect all the super-Feynman and selection rules which select the allowed non-vanishing diagrams. This can be done by performing the null reduction of the relativistic rules in $3+1$ dimensions (see section 3.1 ). In addition, we have to take into account the $\mathrm{U}(1)$ symmetry of the Galilean action (4.3) associated to the mass central charge $M$. This implies that the particle number has to be conserved at each vertex and the only non-vanishing Green functions are the ones whose external particle numbers add up to zero.

\footnotetext{
${ }^{7}$ For details about the null reduction of non-relativistic fermions, see appendix A.

${ }^{8}$ The corresponding computation in component formalism is reported in appendix C.
} 

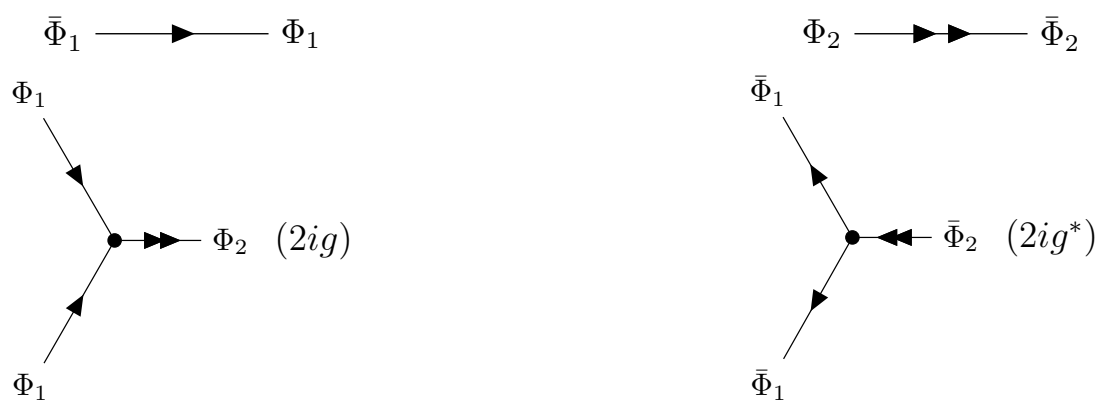

Figure 1. Propagators and vertices in superspace.

\subsection{Super-Feynman and selection rules}

In order to take into account at graphical level the U(1) charge of the two superfields, it is useful to indicate the particle number (or mass) flow by an arrow on the propagator line. As shown in figure 1 we assign a single arrow to $\Phi_{1}$ which has mass $m$ and a double arrow to $\Phi_{2}$ which has mass $2 m$.

The Feynman rules in the non-relativisitic $\mathcal{N}=2$ superspace are:

- Superfield propagators. These are easily obtained from the relativistic ones in eq. (3.7) by replacing $\square \rightarrow 2 i M \partial_{t}+\partial_{i}^{2}$, with $M=m$ or $2 m$ or, in momentum space, $-p^{2} \rightarrow 2 M \omega-\vec{p}^{2}$. We obtain

$$
\left\langle\Phi_{1}(\omega, \vec{p}) \bar{\Phi}_{1}(-\omega,-\vec{p})\right\rangle=i \frac{\delta^{(4)}\left(\theta_{1}-\theta_{2}\right)}{2 m \omega-\vec{p}^{2}+i \varepsilon}, \quad\left\langle\bar{\Phi}_{2}(\omega, \vec{p}) \Phi_{2}(-\omega,-\vec{p})\right\rangle=i \frac{\delta^{(4)}\left(\theta_{1}-\theta_{2}\right)}{4 m \omega-\vec{p}^{2}+i \varepsilon}
$$

As usual in the Galilean setting, we take the energy dimensions as

$$
[\omega]=E^{2}, \quad[\vec{k}]=E, \quad[m]=E^{0}
$$

The propagators for both sectors have a retarded $i \varepsilon$ prescription which follows the order of fields shown in figure 1, where the exchange of particles with anti-particles in sector 2 is manifest from the reversed order of the fields with respect to sector $1 .{ }^{9}$

- Vertices. These are cubic vertices easily read from the action in (4.3). The particle number conservation at each vertex translates into the condition that the numbers of entering and exiting arrows have to match (see figure 1).

Since the null reduction does not affect the grassmannian part of the superspace, supergraphs are built as in the relativistic case. In particular, rules (3.6) still hold, so that we have one extra $\bar{D}^{2}\left(D^{2}\right)$ for each chiral (anti-chiral) superfield entering or exiting

\footnotetext{
${ }^{9}$ In configuration space the $i \varepsilon$ prescription translates into a retarded prescription for the propagator. In fact, the Fourier transform of (5.1) reads $(M=m$ or $2 m)$

$$
D(\vec{x}, t)=\int \frac{d^{2} p d \omega}{(2 \pi)^{3}} i \frac{\delta^{(4)}\left(\theta_{1}-\theta_{2}\right)}{2 M \omega-\vec{p}^{2}+i \varepsilon} e^{-i(\omega t-\vec{p} \cdot \vec{x})}=-\frac{i \Theta(t)}{4 \pi t} e^{i \frac{M \vec{x}^{2}}{2 t}} \delta^{(4)}\left(\theta_{1}-\theta_{2}\right)
$$

where $\Theta$ is the Heaviside function.
} 


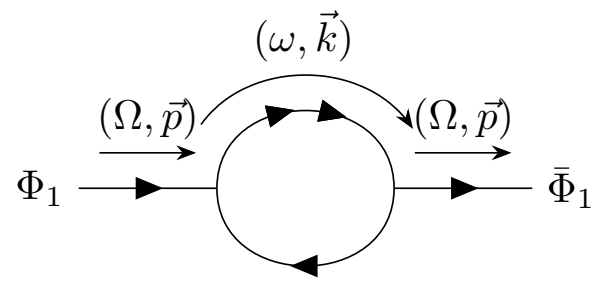

Figure 2. One-loop correction to the self-energy of the $\Phi_{1}$ superfield.

a vertex. The only important difference is that in the present case the grassmannian derivatives are the non-relativistic ones in (2.12). D-algebra can then be performed as summarized in section 3.1 in order to reduce the supergraph to a combination of ordinary Feynman graphs for functions that are local in $(\theta, \bar{\theta})$. In the non-relativistic case identities that are crucial for the D-algebra are still (3.8) (with $D, \bar{D}$ replacing $\mathcal{D}, \overline{\mathcal{D}}$ ), and (see eqs. (A.27), (A.29))

$$
\begin{array}{rlrl}
{\left[D^{\alpha}, \bar{D}^{2}\right]} & =\sqrt{2} M \bar{D}_{1} \delta_{1}^{\alpha}+i\left(\bar{\sigma}^{\mu}\right)^{\alpha \beta} \partial_{\mu} \bar{D}_{\beta}, & & {\left[\bar{D}^{\alpha}, D^{2}\right]=-\sqrt{2} M D_{1} \delta_{1}^{\alpha}-i\left(\bar{\sigma}^{\mu}\right)^{\alpha \beta} \partial_{\mu} D_{\beta},} \\
D^{2} \bar{D}^{2} D^{2} & =\left(2 i M \partial_{t}+\partial_{i}^{2}\right) D^{2}, & \bar{D}^{2} D^{2} \bar{D}^{2}=\left(2 i M \partial_{t}+\partial_{i}^{2}\right) \bar{D}^{2}
\end{array}
$$

where $\mu \in\{+, 1,2\}$.

Since the interaction part of the action still contains cubic vertices as in the relativistic case, the possible topologies of supergraphs are the same (for supergraphs of the ordinary WZ model, see for instance [41, 42]). However, the particle number conservation combined with the analyticity properties leads to extra selection rules that are peculiar of the nonrelativistic models, and drastically reduce the number of non-vanishing diagrams.

First of all, the retarded nature of the non-relativistic scalar propagator, which in momentum space is linear in the energy $\omega$, implies

Selection rule 5.1. Arrows inside a Feynman diagram cannot form a closed loop.

This can be easily seen to be a consequence of the residue theorem in momentum space and is better illustrated with an example. We consider the quantum correction to the self-energy of the superpropagator in sector 1 , as depicted in figure 2 .

This diagram gives the following contribution to the effective action

$$
i \Gamma_{1}^{(2)}\left(\Phi_{1}, \bar{\Phi}_{1}\right)=4|g|^{2} \int d^{4} \theta \frac{d \omega d^{2} k}{(2 \pi)^{3}} \frac{\Phi_{1}(\Omega, \vec{p}, \theta) \bar{\Phi}_{1}(\Omega, \vec{p}, \theta)}{\left[4 m \omega-\vec{k}^{2}+i \varepsilon\right]\left[2 m(\omega-\Omega)-(\vec{k}-\vec{p})^{2}+i \varepsilon\right]}
$$

We can perform the $\omega$ integration first, as the integrand is sufficiently regular to allow the use of the residue theorem. The poles of the integrand

$$
\omega^{(1)}=\frac{\vec{k}^{2}}{4 m}-i \varepsilon, \quad \omega^{(2)}=\Omega+\frac{(\vec{k}-\vec{p})^{2}}{2 m}-i \varepsilon
$$

are both located in the lower-half complex plane, and so we can close the integration contour in the upper half-plane, obtaining

$$
\Gamma_{1}^{(2)}\left(\Phi_{1}, \bar{\Phi}_{1}\right)=0
$$




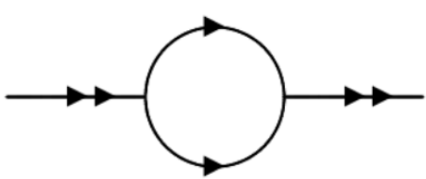

(a)

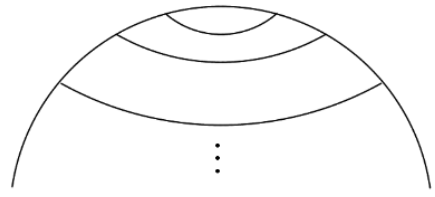

(b)

Figure 3. Configurations allowed (a) and forbidden (b) by selection rule 5.1.

Analogously, in configuration space, the vanishing of the two-point function arises from the product of two Heaviside functions with opposite arguments, which would have support only in one point. By normal ordering, we choose to put this contribution to zero [43].

Since this argument works whenever all the $\omega$ poles are at the same side of the complex $\omega$ plane (i.e. circulating arrows in the loop), selection rule 5.1 holds in general. However, there is an important caveat: the selection rule relies on the possibility to perform the $\omega$-integration by using the residue theorem, which in turn requires the integrand to be sufficiently decreasing at infinity for applying Jordan's lemma. Propagators should guarantee that this is always the case, but $D$-algebra might introduce extra $\omega$ factors as a result of the commutation rules (5.4). As it will be discussed in 5.2, this never happens. As a consequence, selection rule 5.1 is true even before performing D-algebra.

Selection rule 5.1 provides fundamental restrictions that make the non-relativistic case very different from the relativistic one, and in this respect easier to study. For example, an immediate consequence is that at one loop, one-particle irreducible diagrams with two external lines admit only one non-vanishing configuration, the one given in figure 3(a). This rule is true also when the diagram is part of a bigger graph. As a consequence, the topology shown in figure 3(b) is always forbidden, when the number of horizontal lines is bigger than two.

Further selection rules can be obtained from the application of the particle number conservation:

Selection rule 5.2. The (sub)diagrams appearing in figure 4 are forbidden by particle number conservation. Configuration (e) is forbidden only for an even number of horizontal lines on the right side.

This statement can be proved by drawing every possible configuration of arrows and checking that no configurations arise, which respect conservation at all vertices.

As an example, we consider diagram 4(a) for which all possible configurations of arrows are drawn in figure 5. It can be seen that in all the configurations we cannot consistently assign arrows in the top right vertex.

\subsection{Renormalizability of the theory}

Renormalizability in superspace can be investigated by studying the superficial degree of divergence of a generic supergraph with $L$ loops, $E=E_{C}+E_{A}$ external lines, $P$ internal propagators and $V=V_{C}+V_{A}$ vertices, where the $C$ and $A$ subscripts stand for chiral and anti-chiral, respectively. 


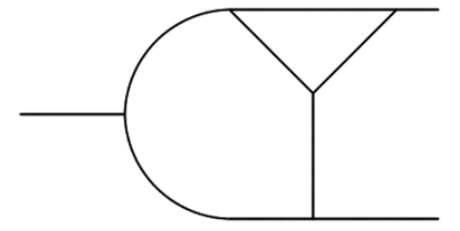

(a)

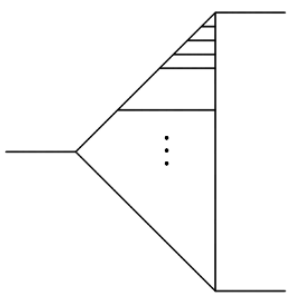

(c)

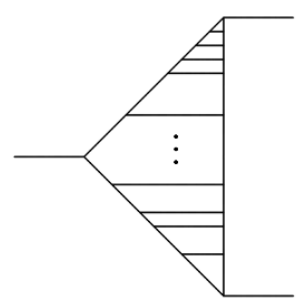

(d)

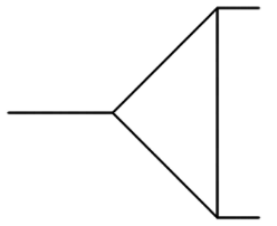

(b)

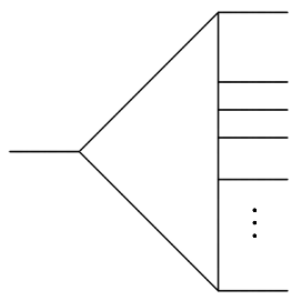

(e)

Figure 4. Set of vanishing (sub)diagrams due to particle number conservation. In (e) the number of horizontal lines on the right side is required to be even.

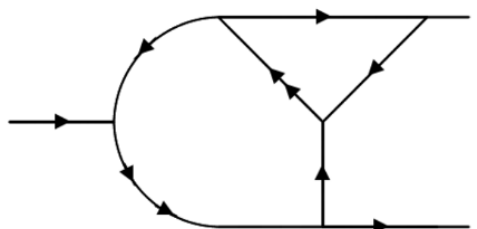

(a)

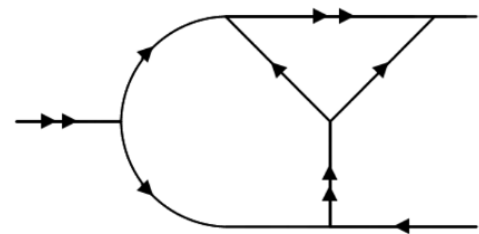

(c)

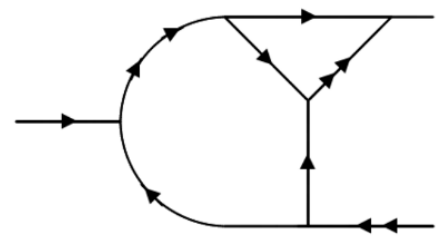

(e)

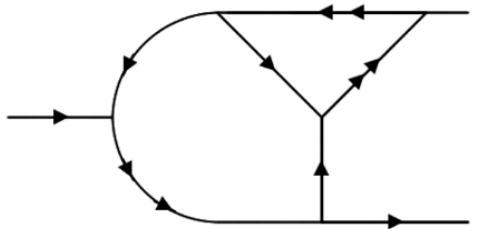

(b)

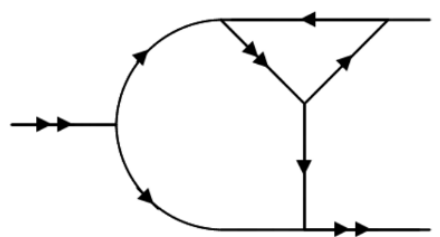

(d)

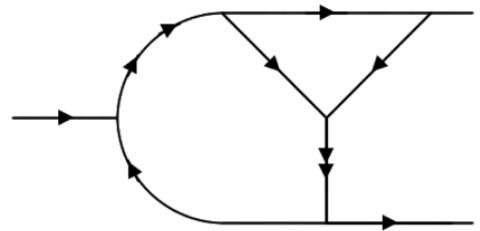

(f)

Figure 5. All possible configurations of arrows in an example of subdiagram. All of them are forbidden by particle number conservation at the upper right vertex.

For a connected graph we can use the topological constraint

$$
L=P-V+1
$$

Since there are only cubic vertices, the relation $E+2 P=3 V$ also holds and when combined 
with the previous constraint leads to

$$
P=E+3 L-3
$$

Finally, in this theory the propagator always connects a chiral vertex with an anti-chiral one. This further restricts the allowed configurations of diagrams, which have to satisfy

$$
3 V_{A}=P+E_{A}, \quad 3 V_{C}=P+E_{C}
$$

The integrand associated to a 1PI supergraph is given by the product of $P$ superpropagators (5.1) times a number of $D, \bar{D}$ derivatives acting on the grassmannian delta functions. Counting the number of these derivatives, we have a factor of $\left(\bar{D}^{2}\right)^{2}$ associated to each internal chiral vertex, a factor of $\left(D^{2}\right)^{2}$ associated to each internal anti-chiral vertex, a factor of $\bar{D}^{2}$ for each chiral vertex with an external line attached, and finally a factor of $D^{2}$ for each anti-chiral vertex with an external line attached. The total number of covariant derivatives is then

$$
\left(D^{2}\right)^{2 V_{A}-E_{A}}\left(\bar{D}^{2}\right)^{2 V_{C}-E_{C}}
$$

On the other hand, D-algebra requires one factor $D^{2} \bar{D}^{2}$ for each loop to contract the integral to a point in the $(\theta, \bar{\theta})$ space. This implies that in non-vanishing diagrams the remaining derivatives

$$
\left(D^{2}\right)^{2 V_{A}-E_{A}-L}\left(\bar{D}^{2}\right)^{2 V_{C}-E_{C}-L}
$$

are traded with powers of loop momenta, according to the D-algebra procedure explained in section 3.1.

Using constraints (5.10), the factor of covariant derivatives associated to the supergraph is given by

$$
\left(D^{2} \bar{D}^{2}\right)^{\frac{2}{3} P-L}\left(D^{2}\right)^{-\frac{E_{A}}{3}}\left(\bar{D}^{2}\right)^{-\frac{E_{C}}{3}}
$$

The total contribution from such a diagram is given by this factor multiplied by $P$ propagators $1 / \triangle$ with $\triangle \equiv 2 M \omega-\vec{k}^{2}$, times $L$ integrations on the loop variables.

Looking at the superficial degree of divergence of the integral, the worst case occurs when identities (5.4) can be used to trade $D^{2} \bar{D}^{2}$ with $\triangle$, which then cancel internal propagators. The corresponding integral reads

$$
\int d \omega_{1} d^{2} k_{1} \ldots d \omega_{L} d^{2} k_{L} \frac{\left(D^{2}\right)^{-\frac{E_{A}}{3}}\left(\bar{D}^{2}\right)^{-\frac{E_{C}}{3}}}{\triangle^{L+\frac{P}{3}}}=\int d \omega_{1} d^{2} k_{1} \ldots d \omega_{L} d^{2} k_{L} \frac{\left(D^{2}\right)^{-\frac{E_{A}}{3}}\left(\bar{D}^{2}\right)^{-\frac{E_{C}}{3}}}{\triangle^{2 L+\frac{E}{3}-1}}
$$

where in the last step eq. (5.9) has been used.

Convergence gets even worse in supergraphs where $E_{A}=E_{C}=E / 2$ and the remaining covariant derivatives also combine into inverse propagators. This gives

$$
\int d \omega_{1} d^{2} k_{1} \ldots d \omega_{L} d^{2} k_{L} \frac{1}{\triangle^{2 L+\frac{E}{2}-1}}
$$

The superficial degree of divergence is $\delta=2-E$. It is always negative for $E \geq 3$ and the corresponding integrals give finite contributions. For self-energy diagrams $(E=2)$ 
logarithmic divergences arise, which can be subtracted by wave-function renormalization. Finally, $E=1$ corresponds to tadpoles, whose prototype is

$$
\int \frac{d \omega d^{2} k}{2 M \omega-\vec{k}^{2}+i \varepsilon}
$$

After performing the $\omega$-integration, we can use dimensional regularization to compute the $\vec{k}$ integral. The result is zero since the integral is dimensionful and cannot depend on any possible scale. The non-relativistic WZ model is then renormalizable.

However, in the non-relativistic case we have to prove a further property of the amplitudes, i.e. that the loop integrals on $\omega_{1}, \ldots, \omega_{L}$ are separately convergent. This requires the integrand to behave at least as $1 / \omega_{i}^{2}$ for a given $\omega_{i}$-integration.

To this end we consider a specific loop $L_{i}$ containing $P_{i}$ propagators. The fact that the inverse of the Galilean propagator is linear in the energy, combined with energy conservation at each vertex implies that the $P_{i}$ propagators provide a power $1 / \omega_{i}^{P_{i}}$. Since in a loop we always have $P_{i} \geq 2$ (tadpoles are zero) the convergence of the $\omega_{i}$-integral is guaranteed, as long as there are no $\omega_{i}$ powers at numerator. However, D-algebra may generate these powers. In the worst situation $D$-derivatives produce factors which cancel completely some propagators, contracting points in momentum space. In any case, this process leads to a loop with at least two propagators, which is sufficient to ensure the convergence of the integral. More generally, adjacent loops which in the relativistic case would lead to overlapping divergences, have an even better convergence in $\omega$.

In conclusion, all the energy integrals are convergent, they do not need to be regularized and we can compute them in the complex plane by using the residue theorem. It is important to stress that this property puts selection rule 5.1 on solid grounds.

\subsection{Loop corrections to the self-energy}

We now study quantum corrections to the WZ model (4.3). In order to deal with divergent momentum integrals we use dimensional regularization within the minimal subtraction scheme. Integrals are computed in $d=2-\epsilon$ and a mass scale $\mu$ is introduced to rescale dimensionful quantities. We define renormalized quantities

$$
\left\{\begin{array}{l}
\Phi_{a}=Z_{a}^{-1 / 2} \Phi_{a}^{(B)}=\left(1-\frac{1}{2} \delta_{a}\right) \Phi_{a}^{(B)} \quad a=1,2 \\
g=\mu^{-\epsilon} Z_{g}^{-1} g^{(B)}=\mu^{-\epsilon}\left(1-\delta_{g}\right) g^{(B)}
\end{array}\right.
$$

and determine counterterms proportional to $\delta_{a}, \delta_{g}$

$$
\mathcal{L}_{\text {ren }}+\int d^{4} \theta\left(\delta_{1} \bar{\Phi}_{1} \Phi_{1}+\delta_{2} \bar{\Phi}_{2} \Phi_{2}\right)+\int d^{2} \theta\left[\mu^{\epsilon} g\left(\delta_{g}+\delta_{1}+\frac{1}{2} \delta_{2}\right) \Phi_{1}^{2} \Phi_{2}\right]+\text { h.c. }
$$

which cancel UV divergences.

One loop. By applying selection rule 5.1 to sector 1, we immediately realize that there are no allowed one-loop diagrams, except for the one depicted in figure 2, which was shown to vanish. As a consequence,

$$
\delta_{1}^{(1 \text { loop })}=0
$$




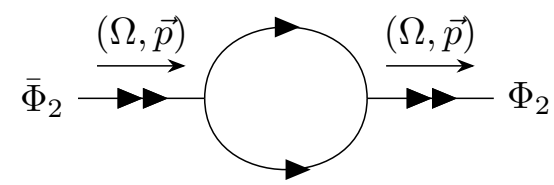

Figure 6. One-loop correction to the self-energy of $\Phi_{2}$.
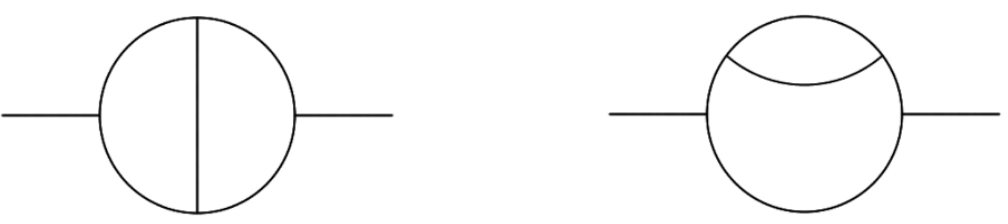

Figure 7. Topologies of possible two-loop corrections to the self-energies.

For the one-loop self-energy in sector 2 we find, instead, the allowed diagram in figure 6 .

After performing D-algebra, the corresponding contribution reads

$$
i \Gamma_{2}^{(2)}\left(\Phi_{2}, \bar{\Phi}_{2}\right)=2|g|^{2} \int d^{4} \theta \frac{d \omega d^{2} k}{(2 \pi)^{3}} \frac{\Phi_{2}(\Omega, \vec{p}, \theta) \bar{\Phi}_{2}(\Omega, \vec{p}, \theta)}{\left[2 m \omega-\vec{k}^{2}+i \varepsilon\right]\left[2 m(\Omega-\omega)-(\vec{p}-\vec{k})^{2}+i \varepsilon\right]}
$$

As already discussed, the $\omega$-integral is convergent and can be easily performed by means of the residue theorem, leading to

$$
\Gamma_{2}^{(2)}\left(\Phi_{2}, \bar{\Phi}_{2}\right)=-\frac{|g|^{2}}{m} \int d^{4} \theta \Phi_{2}(\Omega, \vec{p}, \theta) \bar{\Phi}_{2}(\Omega, \vec{p}, \theta) \int \frac{d^{2} k}{(2 \pi)^{2}} \frac{1}{2 m \Omega-\vec{k}^{2}-(\vec{p}-\vec{k})^{2}+i \varepsilon}
$$

The two-dimensional momentum integral can be now performed using dimensional regularization. Focusing on its divergent part we obtain

$$
\Gamma_{2}^{(2)}\left(\Phi_{2}, \bar{\Phi}_{2}\right) \rightarrow \frac{|g|^{2}}{4 \pi m} \frac{1}{\varepsilon} \int d^{4} \theta \Phi_{2}(\Omega, \vec{p}, \theta) \bar{\Phi}_{2}(\Omega, \vec{p}, \theta)
$$

In the minimal subtraction scheme this leads to the following counterterm

$$
\delta_{2}^{(1 \text { loop })}=-\frac{|g|^{2}}{4 \pi m} \frac{1}{\varepsilon}
$$

Two loops. Selection rules 5.1 and 5.2 are sufficient to rule out any two-loop correction to the self-energies. In fact, looking at the two possible two-loop topologies of diagrams depicted in figure 7 , it is easy to realize that no consistent assignments of arrows exist, or they vanish due to circulating arrows in a loop.

Three loops. At three loops, the set of diagrams is of course richer. In the relativistic setting all possible diagrams have been given in [41], where the three-loop $\beta$-function was computed.

In the non-relativistic case selection rules 5.1 and 5.2 discard almost all possible configurations, leading only to one non-trivial type of diagram, the non-planar one depicted in figure 8. However, looking at all possible assignments of arrows we conclude that there is always a circulating loop, which entails a vanishing result according to selection rule 5.1. Therefore, there are no three-loop corrections to the self-energies of both superfields. 

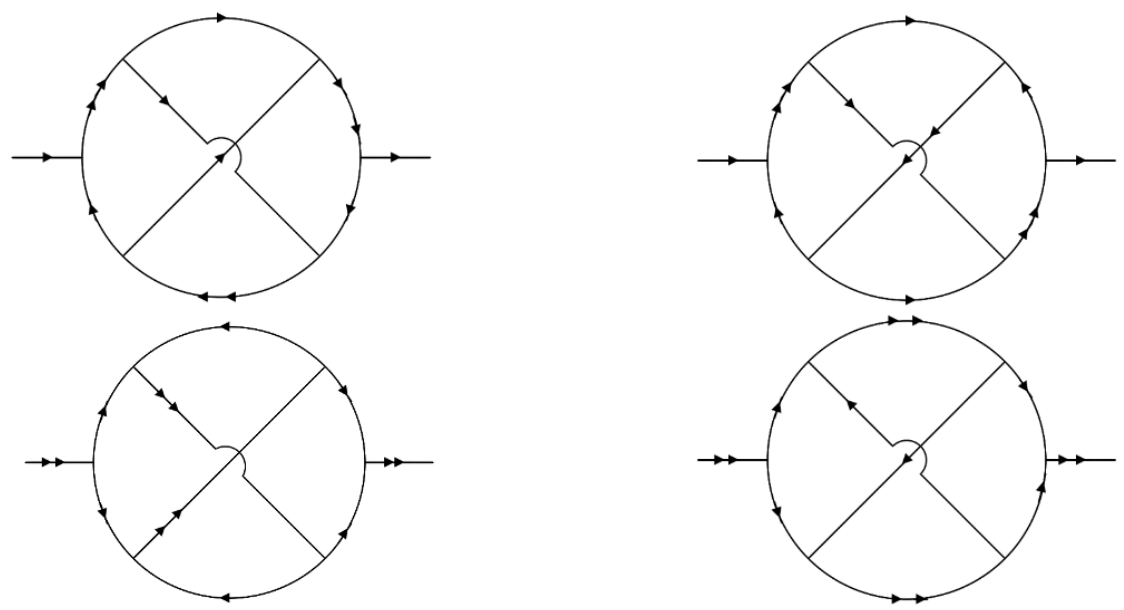

Figure 8. Non-trivial three-loop corrections to the self-energies. We depict all possible assignments of arrows in the lines.

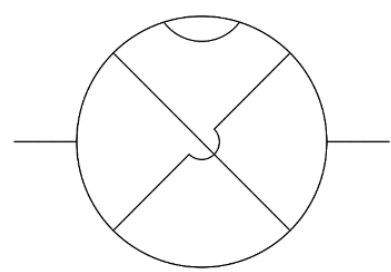

(a)

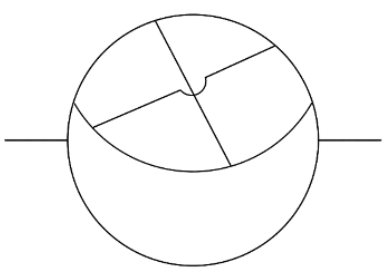

(b)

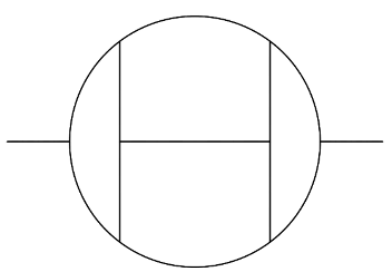

(c)

Figure 9. Non-trivial quantum corrections to the self-energy at four loops.

Four loops. At four loops all possible configurations of self-energy supergraphs can be imported from [42], where the $\beta$-function for the relativistic WZ model was computed.

In the non-relativistic case, once again, the selection rules discard almost all configurations, leaving only the non-trivial diagrams listed in figure 9(a)-(c).

The first two graphs contain as a subgraph the non-planar three-loop diagram already discussed. Therefore, with similar arguments, we can prove that these diagrams vanish. Diagram (c) is a new configuration and in principle it allows for different configurations of arrows depicted in figure 10(a)-(d). It is easy to see that all of them contain circulating loop arrows, thus they vanish by means of selection rule 5.1 .

Higher loops. Up to four loops we have found that non-vanishing quantum corrections to the self-energy appear only in sector 2 and only at one loop. Triggered by these results, the natural question which arises is whether the same pattern repeats at any loop order or we should expect non-vanishing contributions at higher loops.

To face this question, we can start excluding a large class of diagrams which contain recursive structures that can be shown to vanish due to the selection rules. First, all diagrams containing the structures in figure 4 are forbidden by selection rule 5.2. Among the allowed ones we can select for instance the structures in figure 11. Generalizing the previous analysis one can check that all these structures always contain a closed loop of 


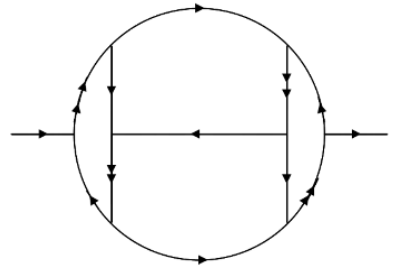

(a)

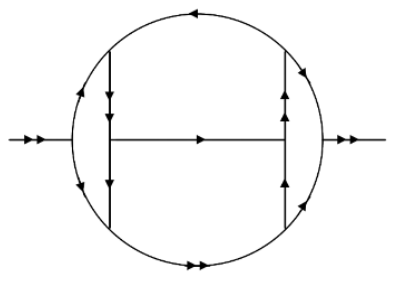

(c)

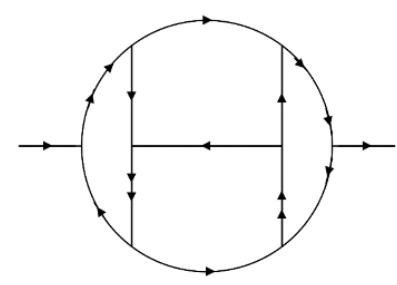

(b)

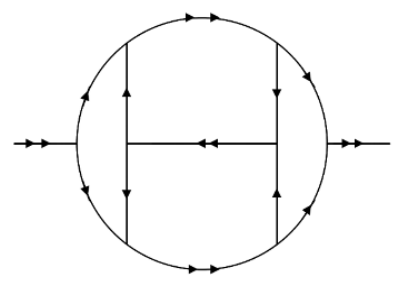

(d)

Figure 10. Allowed assignments of arrows to the lines of diagram (c) in figure 9 .

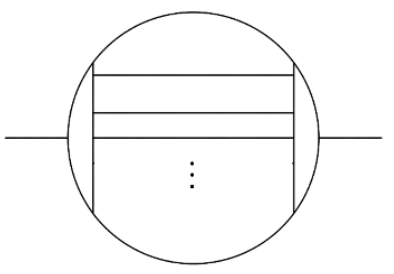

(a)

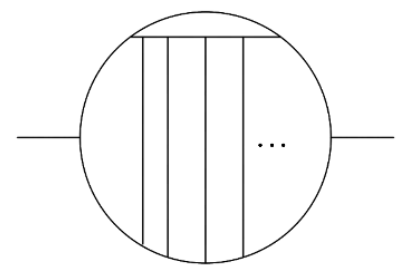

(c)

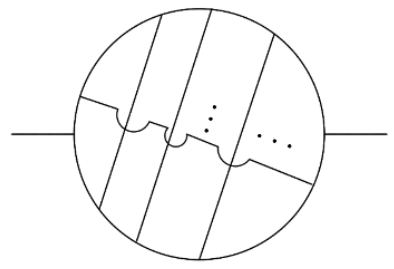

(b)

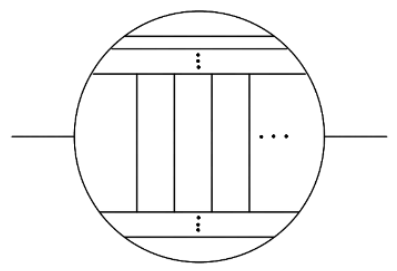

(d)

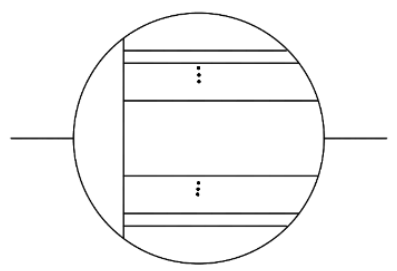

(e)

Figure 11. Non-trivial vanishing quantum corrections to self-energies at generic loop level.

arrows and are eventually ruled out by selection rule 5.1. This remains true for all the diagrams that can be obtained by gluing different structures of figure 11 .

Although these topologies cover a vast number of diagrams, they are not exhaustive and in principle we cannot exclude the appearance of possible non-vanishing contributions from more general configurations, like the one in figure 12. Nonetheless, based on the 


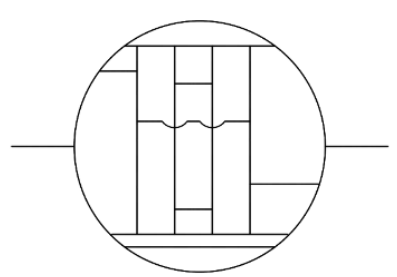

Figure 12. General self-energy diagram.

experience gained up to four loops, we expect that when the numbers of loops increases it becomes more and more difficult to realize configurations of arrows without closed loops. Therefore, we can quite safely conjecture that the self-energy of the $\Phi_{1}$ superfield is not corrected at quantum level, while the one for $\Phi_{2}$ is one-loop exact.

Independently of the validity of this conjecture, it is a matter of fact that in the nonrelativistic model self-energy corrections are much simpler than their $4 \mathrm{~d} \mathcal{N}=1$ relativistic cousins. In fact, in the relativistic case the kinetic term acquires UV divergent corrections at any loop order. Instead, in the non-relativistic model the particle number conservation and the structure of the propagators drastically reduce the number of non-vanishing contributions, leading to a theory which is one-loop exact. In particular, this shows that at quantum level the non-relativistic three-dimensional $\mathcal{N}=2 \mathrm{WZ}$ model cannot be obtained simply from null reduction of the four-dimensional relativistic model.

\subsection{Loop corrections to the vertices}

As already discussed in section 5.2 for a number of external legs equal or greater than three we do not expect UV divergent contributions. Moreover, given that the null reduction does not affect the spinorial part of superspace, we do not expect chiral integrals to be produced. Therefore, the perturbartive non-renormalization theorem for the superpotential should still work. As a consequence, the following constraint on the renormalization functions (5.17) follows

$$
\delta_{g}+\delta_{1}+\frac{1}{2} \delta_{2}=0 \quad \Rightarrow \quad \delta_{g}^{(1 \text { loop })}=\frac{|g|^{2}}{8 \pi m} \frac{1}{\epsilon}
$$

It is worth discussing the three-vertex diagrams anyway in order to investigate how the selection rules restrict the number of possible quantum corrections for configurations with three external fields.

As in the relativistic case, at one-loop there is no way to draw any three-point diagram as long as the model is massless.

At two loops the only supergraph allowed by particle number conservation is the one in figure 13, where all possible configurations of arrows have been depicted. In all the diagrams we see that a circulating loop of arrows appears, thus this diagram is ruled out by selection rule 5.1. In appendix D we present details of the calculation.

Extending this analysis at higher loops, again we find that in the non-relativistic model the number of (finite) quantum contributions is drastically reduced compared to the relativistic case. 


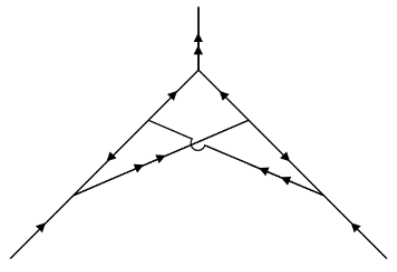

(a)

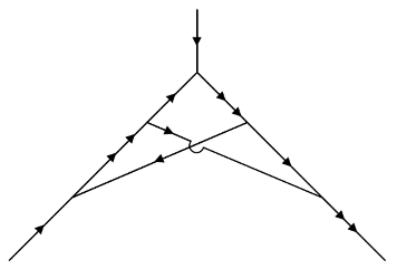

(c)

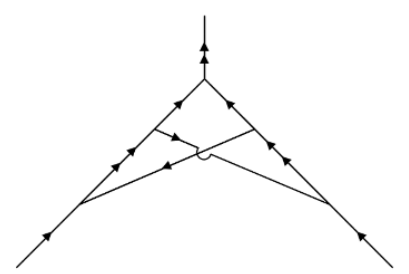

(b)

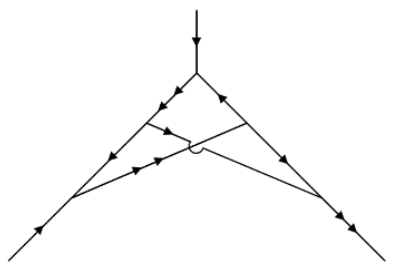

(d)

Figure 13. Two-loop 1PI diagram for the three-point vertex. We depicted all the configurations of arrows associated to the lines.

\subsection{Non-relativistic non-renormalization theorem}

Strong support to the conjectured absence of corrections to the three-point vertex comes from the existence of a non-relativistic version of the non-renormalization theorem. Here we argue that such a theorem can be easily inherited from the relativistic one.

We consider a generic Galilean WZ model for $n$ chiral superfields

$$
S=\int d^{3} x d^{2} \theta d^{2} \bar{\theta} \bar{\Phi}_{a} \Phi_{a}+g \int d^{3} x d^{2} \theta W\left(\Phi_{a}\right)+\text { h.c. }
$$

obtained by null reduction of the relativistic one in eq. (3.14).

The same argument used in the relativistic case can be adapted here in order to rule out quantum corrections to the F-term. As in eq. (3.15) we can introduce one extra chiral superfield $Y$ multiplying the superpotential, which can be set equal to 1 in order to reproduce eq. (5.25), together with wave function renormalization superfields $Z_{a b}$

$$
\tilde{S}=\int d^{3} x d^{4} \theta Z_{a b} \bar{\Phi}_{a} \Phi_{b}+\int d^{3} x d^{2} \theta Y W\left(\Phi_{a}\right)+\text { h.c. }
$$

Since the non-relativistic limit via null reduction technique does not modify the grassmannian part of the superfields in the action, R-symmetry works in the same way as in the relativistic case. Therefore, as in the relativistic case, we assign R-charges $R\left(\Phi_{a}\right)=0$ and $R(Y)=2$.

The regularization that we used, which corresponds to first performing the regular $\omega$ integrals and then the $\vec{k}$-integrals in dimensional regularization, preserves SUSY. Therefore, the Wilsonian effective action at a given scale $\lambda$ will have the following general structure

$$
\tilde{S}_{\lambda}=\int d^{3} x d^{4} \theta K\left(\bar{\Phi}_{a} \Phi_{a}, Z_{a b}, Y, \bar{Y}, D\right)+\int d^{3} x d^{2} \theta W_{\lambda}\left(\Phi_{a}, Y\right)
$$

Now, R-invariance and holomorphicity of the superpotential, combined with the weak coupling limit, give as in the relativistic case $W_{\lambda}=Y W\left(\Phi_{a}\right)$. 


\section{Conclusions}

In this paper we studied the renormalization properties of a $2+1$ dimensional model with $\mathcal{S}_{2} \mathcal{G}$ Galilean SUSY invariance, obtained by null reduction of the four-dimensional relativistic Wess-Zumino model (see eq. (4.1)). The model contains two chiral superfields and it is the simplest theory with F-term interactions compatible with the non-relativistic $\mathrm{U}(1)$ mass invariance. When reduced in components, after integrating out the auxiliary fields, the interaction lagrangian includes cubic derivative and quartic non-derivative local interactions.

We constructed the $\mathcal{N}=2$ non-relativistic superspace as null reduction of the fourdimensional $\mathcal{N}=1$ relativistic one, and quantized the system in this framework. Since null reduction does not affect the grassmanian coordinates and SUSY is totally preserved, loop corrections to the cubic vertex obey a non-relativistic analog of the non-renormalization theorem for the F-terms of the parent relativistic theory.

More interestingly, in the non-relativistic case, U(1) invariance and the retarded nature of the field propagators lead to further selection rules that forbid many topologies of potentially divergent diagrams. As a result, up to four loops, quantum corrections to the Kähler potential are all vanishing with the exception of the one-loop contribution to the $\Phi_{2}$ propagator.

Extending the investigation to higher loops we have provided strong evidence that the combination of the non-renormalization properties of the F-terms and the selection rules, in particular the vanishing of loop diagrams whose arrows form a closed loop, suppresses Galilean UV divergences in a very efficient way and makes the model one-loop exact. Therefore, the non-relativistic model has peculiar quantum properties that render it very different from its relativistic counterpart. At the quantum level, it cannot be simply obtained by null reduction of the ordinary WZ model in $3+1$ dimensions.

The result we have found is reminiscent of relativistic gauge theories with extended SUSY, like for instance the relativistic $\mathcal{N}=2 \mathrm{SYM}$ in $3+1$ dimensions. In that case extended supersymmetry constrains the corrections to the Kähler potential to be related to the F-terms, which are protected by the non-renormalization theorem. In the nonrelativistic model discussed in this paper, instead the protection of the Kähler potential is related to the $\mathrm{U}(1)$ charge conservation at each vertex, which in many diagrammatic contributions constrains arrows to form a closed loop, so leading to a vanishing integral. It would be interesting to investigate if a common hidden mechanism exists, which is responsible of the similar mild UV behavior of these two rather different classes of theories.

At the classical level the model is scale-invariant. However, this symmetry is broken by anomalous quantum corrections. As follows from our result (5.24) the beta function for the coupling $g$ is given by

$$
\beta_{g}=\frac{d g}{d \log \mu}=\frac{g^{3}}{4 \pi m}
$$

and the theory is infrared free at low energies, like the model studied in [43]. If the Kähler potential is one-loop exact, as it seems to be the case, then equation (6.1) is exact. Note that in the single field example without SUSY studied in [43] the beta function is also one- 
loop exact, but for different reasons. There the propagator has no quantum corrections and all the UV divergences come from the vertex corrections.

As a continuation of the study of non-relativistic conformal anomalies in curved Newton-Cartan (NC) background [44-50], it would be interesting to study superconformal anomalies of a Galilean SUSY theory in the presence of a classical NC supergravity source. We leave this as a topic for further work.

A non-relativistic theory of a chiral superfield coupled to a Chern-Simons gauge field, which is invariant under the conformal extension of the $\mathcal{S}_{2} \mathcal{G}$ algebra, was constructed in [25]. We expect that further examples of $\mathcal{S}_{2} \mathcal{G}$ theories may be constructed by coupling the F-term interacting theory discussed in this paper to a Chern-Simons gauge field. These examples should contain trilinear derivative couplings between scalars and fermions and then should be different from existing constructions of non-relativistic SUSY Chern Simons theory built from the $c \rightarrow \infty$ non relativistic limit (see e.g. [51-55]). In analogy to the nonSUSY example studied in [56], we expect that for special values of the F-term coupling $g$ the resulting theory is conformal. We leave a detailed study as a topic for further investigation. These extensions may provide a useful theoretical SUSY setting for studying non-abelian anyons [55, 57] and non-relativistic particle-vortex dualities [58].

\section{Acknowledgments}

This work has been supported in part by Italian Ministero dell'Istruzione, Università e Ricerca (MIUR), and by Istituto Nazionale di Fisica Nucleare (INFN) through the "Gauge and String Theory" (GAST) and "Gauge Theories, Strings, Supergravity" (GSS) research projects.

\section{A Conventions}

In this appendix we collect SUSY conventions in $3+1$ and $2+1$ dimensions. For conventions in four dimensions we primarily refer to [59].

Spinors. In $3+1$ dimensional Minkowski space-time we take the metric $\eta^{M N}=$ $\operatorname{diag}(-1,1,1,1)$ and denote left-handed Weyl spinors as $\psi_{\alpha}$, while right-handed ones as $\bar{\chi}^{\dot{\alpha}}$.

Spinorial indices are raised and lowered as

$$
\psi^{\alpha}=\epsilon^{\alpha \beta} \psi_{\beta}, \quad \bar{\chi}_{\dot{\alpha}}=\epsilon_{\dot{\alpha} \dot{\beta}} \bar{\chi}^{\dot{\beta}}
$$

where the Levi-Civita symbol is chosen to be

$$
\epsilon^{\alpha \beta}=\epsilon^{\dot{\alpha} \dot{\beta}}=-\epsilon_{\alpha \beta}=-\epsilon_{\dot{\alpha} \dot{\beta}}=\left(\begin{array}{cc}
0 & 1 \\
-1 & 0
\end{array}\right)
$$

Contractions of spinorial quantities are given by

$$
\chi \cdot \psi=\chi^{\alpha} \psi_{\alpha}=\psi \cdot \chi, \quad \bar{\chi} \cdot \bar{\psi}=\bar{\chi}_{\dot{\alpha}} \bar{\psi}^{\dot{\alpha}}=\bar{\psi} \cdot \bar{\chi}
$$


Complex conjugation changes the chirality of spinors. The prescription for the signs is

$$
\left(\psi^{\alpha}\right)^{\dagger}=\bar{\psi}^{\dot{\alpha}}, \quad\left(\psi_{\alpha}\right)^{\dagger}=\bar{\psi}_{\dot{\alpha}}, \quad\left(\bar{\chi}^{\dot{\alpha}}\right)^{\dagger}=\chi^{\alpha}, \quad\left(\bar{\chi}_{\dot{\alpha}}\right)^{\dagger}=\chi_{\alpha}
$$

We use sigma matrices

$$
\sigma^{M}=\left(\mathbf{1}, \sigma^{i}\right), \quad \bar{\sigma}^{M}=\left(\mathbf{1},-\sigma^{i}\right)
$$

where we have defined $\left(\bar{\sigma}^{M}\right)^{\dot{\alpha} \alpha}=\epsilon^{\dot{\alpha} \dot{\beta}} \epsilon^{\alpha \beta}\left(\sigma^{M}\right)_{\beta \dot{\beta}}$. They satisfy the following set of useful identities

$$
\begin{aligned}
\left(\sigma^{M}\right)_{\alpha \dot{\alpha}}\left(\bar{\sigma}_{M}\right)^{\dot{\beta} \beta} & =-2 \delta_{\alpha}{ }^{\beta} \delta_{\dot{\alpha}}^{\dot{\beta}}, \quad\left(\sigma^{M}\right)_{\alpha \dot{\alpha}}\left(\sigma_{M}\right)_{\beta \dot{\beta}}=-2 \epsilon_{\alpha \beta} \epsilon_{\dot{\alpha} \dot{\beta}}, \quad \operatorname{Tr}\left(\sigma^{M} \bar{\sigma}^{N}\right)=-2 \eta^{M N}, \\
\left(\sigma^{M} \bar{\sigma}^{N}+\sigma^{N} \bar{\sigma}^{M}\right)_{\alpha}^{\beta} & =-2 \eta^{M N} \delta_{\alpha}{ }^{\beta}, \quad\left(\bar{\sigma}^{M} \sigma^{N}+\bar{\sigma}^{N} \sigma^{M}\right)_{\dot{\alpha}}^{\dot{\beta}}=-2 \eta^{M N} \delta_{\dot{\alpha}}^{\dot{\beta}} \quad \text { (A.6) }
\end{aligned}
$$

Spinorial derivatives. In order to manipulate expressions with spinorial objects it is useful to adopt a notation where spinorial indices are manifest. For the case of vectors and in particular for partial derivatives this is achieved by defining

$$
\partial_{\alpha \dot{\alpha}}=\left(\sigma^{M}\right)_{\alpha \dot{\alpha}} \partial_{M}, \quad \partial^{\alpha \dot{\alpha}}=\epsilon^{\alpha \beta} \epsilon^{\dot{\alpha} \dot{\beta}} \partial_{\beta \dot{\beta}}=\left(\bar{\sigma}^{M}\right)^{\dot{\alpha} \alpha} \partial_{M}, \quad \partial_{M}=-\frac{1}{2}\left(\bar{\sigma}_{M}\right)^{\dot{\alpha} \alpha} \partial_{\alpha \dot{\alpha}}
$$

which in particular imply

$$
\square \equiv \partial^{M} \partial_{M}=-\frac{1}{2} \partial^{\alpha \dot{\alpha}} \partial_{\alpha \dot{\alpha}}, \quad \partial^{\alpha \dot{\gamma}} \partial_{\dot{\gamma} \beta}=-\delta_{\beta}^{\alpha} \square
$$

We assign rules for the coordinates consistently with the requirement $\partial_{M} x^{M}=\partial_{\alpha \dot{\alpha}} x^{\alpha \dot{\alpha}}=4$, that is

$$
x^{\alpha \dot{\alpha}}=-\frac{1}{2}\left(\bar{\sigma}_{M}\right)^{\dot{\alpha} \alpha} x^{M}, \quad x^{M}=\left(\sigma^{M}\right)_{\alpha \dot{\alpha}} x^{\alpha \dot{\alpha}}
$$

It follows that $x^{2} \equiv x^{M} x_{M}=-2 x^{\alpha \dot{\alpha}} x_{\alpha \dot{\alpha}}$.

Finally, we define partial spinorial derivatives acting on Grassmann variables as

$$
\partial_{\alpha} \theta^{\beta}=\delta_{\alpha}{ }^{\beta}, \quad \partial^{\beta} \theta_{\alpha}=-\delta_{\alpha}{ }^{\beta}, \quad \bar{\partial}_{\dot{\alpha}} \bar{\theta}^{\dot{\beta}}=\delta_{\dot{\alpha}}^{\dot{\beta}}, \quad \bar{\partial}^{\dot{\beta}} \bar{\theta}_{\dot{\alpha}}=-\delta_{\dot{\beta}}^{\dot{\alpha}}
$$

Imposing the reality of $\delta_{M}^{N}=\left[\partial_{M}, x^{N}\right]$ and $\delta_{\alpha}^{\beta}=\left\{\partial_{\alpha}, \theta^{\beta}\right\}$ we find that spacetime derivatives are anti-hermitian, $\left(\partial_{M}\right)^{\dagger}=-\partial_{M}$, while the spinorial ones are hermitian, $\left(\partial_{\alpha}\right)^{\dagger}=\bar{\partial}_{\dot{\alpha}}$.

Superspace. The SUSY generators can be written as

$$
P_{\alpha \dot{\alpha}}=-i \partial_{\alpha \dot{\alpha}}, \quad \mathcal{Q}_{\alpha}=i\left(\partial_{\alpha}+\frac{i}{2} \bar{\theta}^{\dot{\alpha}} \partial_{\alpha \dot{\alpha}}\right), \quad \overline{\mathcal{Q}}_{\dot{\alpha}}=-i\left(\bar{\partial}_{\dot{\alpha}}+\frac{i}{2} \theta^{\alpha} \partial_{\alpha \dot{\alpha}}\right)
$$

such that the algebra is $\left\{\mathcal{Q}_{\alpha}, \overline{\mathcal{Q}}_{\dot{\alpha}}\right\}=i \partial_{\alpha \dot{\alpha}}=-P_{\alpha \dot{\alpha}}$. The covariant differential operators which anticommute with the supercharges are

$$
\mathcal{D}_{\alpha}=\partial_{\alpha}-\frac{i}{2} \bar{\theta}^{\dot{\alpha}} \partial_{\alpha \dot{\alpha}}=-i \mathcal{Q}_{\alpha}-i \bar{\theta}^{\dot{\alpha}} \partial_{\alpha \dot{\alpha}}, \quad \overline{\mathcal{D}}_{\dot{\alpha}}=\bar{\partial}_{\dot{\alpha}}-\frac{i}{2} \theta^{\alpha} \partial_{\alpha \dot{\alpha}}=i \overline{\mathcal{Q}}_{\dot{\alpha}}-i \theta^{\alpha} \partial_{\alpha \dot{\alpha}}
$$


and they satisfy

$$
\left\{\mathcal{D}_{\alpha}, \overline{\mathcal{D}}_{\dot{\alpha}}\right\}=-i \partial_{\alpha \dot{\alpha}}=P_{\alpha \dot{\alpha}}
$$

With these conventions, we obtain

$$
\overline{\mathcal{Q}}_{\dot{\alpha}}=\left(\mathcal{Q}_{\alpha}\right)^{\dagger}, \quad \overline{\mathcal{D}}_{\dot{\alpha}}=\left(\mathcal{D}_{\alpha}\right)^{\dagger}
$$

We define

$$
\mathcal{Q}^{2} \equiv \frac{1}{2} \mathcal{Q}^{\alpha} \mathcal{Q}_{\alpha}, \quad \overline{\mathcal{Q}}^{2} \equiv \frac{1}{2} \overline{\mathcal{Q}}_{\dot{\alpha}} \overline{\mathcal{Q}}^{\dot{\alpha}}, \quad \mathcal{D}^{2} \equiv \frac{1}{2} \mathcal{D}^{\alpha} \mathcal{D}_{\alpha}, \quad \overline{\mathcal{D}}^{2} \equiv \frac{1}{2} \overline{\mathcal{D}}_{\dot{\alpha}} \overline{\mathcal{D}}^{\dot{\alpha}}
$$

Chiral superfields. Chiral superfields satisfy $\overline{\mathcal{D}}_{\dot{\alpha}} \Sigma\left(x^{M}, \theta^{\alpha}, \bar{\theta}^{\dot{\alpha}}\right)=0$, and can be written as

$$
\Sigma\left(x_{L}, \theta, \bar{\theta}\right)=\phi\left(x_{L}\right)+\theta^{\alpha} \psi_{\alpha}\left(x_{L}\right)-\theta^{2} F\left(x_{L}\right), \quad x_{L}^{\alpha \dot{\alpha}} \equiv x^{\alpha \dot{\alpha}}-i \theta^{\alpha} \bar{\theta}^{\dot{\alpha}}
$$

Similarly, anti-chiral superfields satisfy $\mathcal{D}_{\alpha} \bar{\Sigma}\left(x^{M}, \theta^{\alpha}, \bar{\theta}^{\dot{\alpha}}\right)=0$, whose solution is

$$
\bar{\Sigma}\left(x_{R}, \theta, \bar{\theta}\right)=\bar{\phi}\left(x_{R}\right)+\bar{\theta}_{\dot{\alpha}} \bar{\psi}^{\dot{\alpha}}\left(x_{R}\right)-\bar{\theta}^{2} \bar{F}\left(x_{R}\right), \quad x_{R}^{\alpha \dot{\alpha}} \equiv x^{\alpha \dot{\alpha}}+i \theta^{\alpha} \bar{\theta}^{\dot{\alpha}}
$$

Using definitions $\theta^{2} \equiv \frac{1}{2} \theta^{\alpha} \theta_{\alpha}, \bar{\theta}^{2} \equiv \frac{1}{2} \bar{\theta}_{\dot{\alpha}} \bar{\theta}^{\dot{\alpha}}$, we find the following compact expression for the components of a chiral superfield

$$
\phi=\Sigma\left|, \quad \psi_{\alpha}=\mathcal{D}_{\alpha} \Sigma\right|, \quad F=\mathcal{D}^{2} \Sigma \mid
$$

where $\mid$ means that we evaluate the expression at $\theta=\bar{\theta}=0$. The anti-chiral components are simply given by the complex conjugated of these expressions.

Pauli matrices in light-cone coordinates. Pauli matrices matrices in light-cone coordinates are

$$
\begin{array}{ll}
\sigma^{ \pm}=\frac{1}{\sqrt{2}}\left(\sigma^{3} \pm \sigma^{0}\right), & \bar{\sigma}^{ \pm}=\frac{1}{\sqrt{2}}\left(\bar{\sigma}^{3} \pm \bar{\sigma}^{0}\right) \\
\sigma^{-}=-\bar{\sigma}^{+}=\sqrt{2}\left(\begin{array}{cc}
0 & 0 \\
0 & -1
\end{array}\right), & \sigma^{+}=-\bar{\sigma}^{-}=\sqrt{2}\left(\begin{array}{ll}
1 & 0 \\
0 & 0
\end{array}\right)
\end{array}
$$

Therefore, for instance we write (from (A.7))

$$
\begin{aligned}
& \partial_{\alpha \dot{\alpha}}=\left(\sigma^{+}\right)_{\alpha \dot{\alpha}} \partial_{+}+\left(\sigma^{-}\right)_{\alpha \dot{\alpha}} \partial_{-}+\left(\sigma^{1}\right)_{\alpha \dot{\alpha}} \partial_{1}+\left(\sigma^{2}\right)_{\alpha \dot{\alpha}} \partial_{2} \\
& \partial^{\alpha \dot{\alpha}}=\left(\bar{\sigma}^{+}\right)^{\dot{\alpha} \alpha} \partial_{+}+\left(\bar{\sigma}^{-}\right)^{\dot{\alpha} \alpha} \partial_{-}+\left(\bar{\sigma}^{1}\right)^{\dot{\alpha} \alpha} \partial_{1}+\left(\bar{\sigma}^{2}\right)^{\dot{\alpha} \alpha} \partial_{2}
\end{aligned}
$$

with $\partial_{ \pm}=\frac{1}{\sqrt{2}}\left(\partial_{3} \pm \partial_{0}\right)$ 
Conventions in $\mathbf{2}+\mathbf{1}$ dimensions. Non-relativistic quantities in $2+1$ dimensions are obtained from the null reduction of $3+1$ dimensional Minkowski spacetime. The prescription is to introduce light-cone coordinates $x^{M}=\left(x^{-}, x^{+}, x^{1}, x^{2}\right)=\left(x^{-}, x^{\mu}\right)$, compactify along a small circle in the $x^{-}$direction and perform the identifications

$$
\partial_{-} \rightarrow i m, \quad \partial_{+} \rightarrow \partial_{t}, \quad \phi\left(x^{M}\right)=e^{i m x^{-}} \varphi\left(x^{\mu}\right)
$$

where $m$ is the adimensional eigenvalue of the $\mathrm{U}(1)$ mass operator and $\phi\left(x^{M}\right)$ is a local field.

Non-relativistic fermions in $2+1$ dimensions are parametrized by two complex Grassmann scalars $\xi\left(x^{\mu}\right)$ and $\chi\left(x^{\mu}\right)$. Under null reduction the identification with the $3+1$ dimensional left-handed Weyl spinor $\psi\left(x^{M}\right)$ works as follows

$$
\psi_{\alpha}\left(x^{M}\right)=e^{i m x^{-}} \tilde{\psi}_{\alpha}\left(x^{\mu}\right)=e^{i m x^{-}}\left(\begin{array}{c}
\xi\left(x^{\mu}\right) \\
\chi\left(x^{\mu}\right)
\end{array}\right)
$$

Under complex conjugation we choose the prescription

$$
\bar{\psi}_{\dot{\alpha}}=\left(\psi_{\alpha}\right)^{\dagger}=e^{-i m x^{-}}\left(\tilde{\psi}_{\alpha}\right)^{\dagger} \equiv e^{-i m x^{-}}\left(\begin{array}{c}
\bar{\xi}\left(x^{\mu}\right) \\
\bar{\chi}\left(x^{\mu}\right)
\end{array}\right)
$$

Using identities (A.1) it is easy to infer the identification with the components of $\psi^{\alpha}$ and $\bar{\psi}^{\dot{\alpha}}$.

Taking the mass as a dimensionless parameter enforces the energy dimensions of the non-relativistic fermion to be

$$
[\xi]=E^{2}, \quad[\chi]=E
$$

These assignments immediately follow when performing the null reduction of the Weyl Lagrangian

$$
\mathcal{L}=i \psi^{\dagger} \bar{\sigma}^{M} \partial_{M} \psi \rightarrow \sqrt{2} m \bar{\xi} \xi+\sqrt{2} i \bar{\chi} \partial_{t} \chi-i \bar{\xi}\left(\partial_{1}-i \partial_{2}\right) \chi-i \bar{\chi}\left(\partial_{1}+i \partial_{2}\right) \xi
$$

We observe that the only dynamical component is $\chi$, while $\xi$ turns out to be an auxiliary field that can be integrated out from the action.

Since null reduction affects only space-time coordinates without modifying the spinorial ones, we obtain $\mathcal{N}=2$ supersymmetry in three dimensions. According to the ordinary pattern for which the three dimensional $\mathcal{N}=2$ superspace is "equal" to the four-dimensional $\mathcal{N}=1$ superspace, all the properties related to manipulations of covariant derivatives and supercharges, e.g. the D-algebra procedure, are directly inherited from the $(3+1)$ relativistic theory under a suitable re-interpretation of the spinorial objects.

In particular, the algebra of covariant derivatives reads

$$
\left\{D_{\alpha}, \bar{D}_{\beta}\right\}=-i \partial_{\alpha \beta}, \quad\left\{D^{\alpha}, \bar{D}^{\beta}\right\}=-i \partial^{\beta \alpha}
$$

where, as follows from (A.20), the three-dimensional derivatives are given by

$$
\partial_{\alpha \beta}=\left(\begin{array}{cc}
\sqrt{2} \partial_{t} & \partial_{1}-i \partial_{2} \\
\partial_{1}+i \partial_{2}-i \sqrt{2} M
\end{array}\right) \quad \partial^{\alpha \beta}=\left(\begin{array}{cc}
-i \sqrt{2} M & -\left(\partial_{1}-i \partial_{2}\right) \\
-\left(\partial_{1}+i \partial_{2}\right) & \sqrt{2} \partial_{t}
\end{array}\right)
$$


They satisfy the following identities

$$
\partial^{\alpha \beta}=\epsilon^{\alpha \delta} \epsilon^{\beta \gamma} \partial_{\gamma \delta} \quad \partial_{\beta \alpha}=\epsilon_{\alpha \gamma} \epsilon_{\beta \delta} \partial^{\gamma \delta}
$$

Therefore, we have for instance $\bar{\xi}_{\alpha} \partial^{\alpha \beta} \chi_{\beta}=\bar{\xi}^{\alpha} \partial_{\beta \alpha} \chi^{\beta}$. Identities which turn out to be useful for the reduction of the action to components are

$$
\begin{aligned}
& {\left[D^{\alpha}, \bar{D}^{2}\right]=i \partial^{\beta \alpha} \bar{D}_{\beta}, \quad\left[\bar{D}^{\alpha}, D^{2}\right]=-i \partial^{\alpha \beta} D_{\beta}} \\
& D^{2} \bar{D}^{2}+\bar{D}^{2} D^{2}=\left(2 i M \partial_{t}+\partial_{i}^{2}\right)+D^{\alpha} \bar{D}^{2} D_{\alpha}=\left(2 i M \partial_{t}+\partial_{i}^{2}\right)+\bar{D}_{\alpha} D^{2} \bar{D}^{\alpha}
\end{aligned}
$$

\section{B Non-relativistic Wess-Zumino model in components}

In this appendix we perform the reduction to components of the non-relativistic superaction (4.3) and show how to obtain expression (4.8) after eliminating the auxiliary fields.

For simplicity we focus only on the Kahler term. Applying the prescription for the Berezin integration (2.18) we can write

$$
S_{\text {kin }}=\int d^{3} x\left[\bar{D}^{2} \bar{\Sigma}_{a} D^{2} \Sigma_{a}+\bar{D}_{\alpha} \bar{\Sigma}_{a} \bar{D}^{\alpha} D^{2} \Sigma_{a}+\bar{\Sigma}_{a} \bar{D}^{2} D^{2} \Sigma_{a}\right]
$$

where the non-relativistic covariant derivatives are given in eq. (2.12) and $a=1,2$ labels the two sectors of superfields.

We define the theta expansion of the Wess-Zumino chiral superfields as (here $\theta^{1}, \theta^{2}$ indicate components 1 and 2 of the $\theta^{\alpha}$ spinor)

$$
\begin{aligned}
& \Sigma_{1}=\varphi_{1}+\theta^{1} \xi_{1}+\theta^{2} 2^{\frac{1}{4}} \sqrt{m} \chi_{1}-\frac{1}{2} \theta^{\alpha} \theta_{\alpha} F_{1} \\
& \Sigma_{2}=\varphi_{2}+\theta^{1} \xi_{2}+\theta^{2} i 2^{\frac{1}{4}} \sqrt{2 m} \chi_{2}-\frac{1}{2} \theta^{\alpha} \theta_{\alpha} F_{2}
\end{aligned}
$$

where a convenient rescaling of the grassmannian fields has been implemented in order to have the standard normalization of the kinetic terms, with $\varphi_{a}$ and $\chi_{a}$ sharing the same dimensions. Using these conventions we obtain

$$
\begin{aligned}
S_{\text {kin }}= & \int d^{3} x\left[2 i m \bar{\varphi}_{1}\left(\partial_{t} \varphi_{1}\right)+\bar{\varphi}_{1} \partial_{i}^{2} \varphi_{1}-4 i m \bar{\varphi}_{2}\left(\partial_{t} \varphi_{2}\right)+\bar{\varphi}_{2} \partial_{i}^{2} \varphi_{2}+\bar{F}_{1} F_{1}+\bar{F}_{2} F_{2}\right. \\
& +\sqrt{2} m \bar{\xi}_{1} \xi_{1}+2 i m \bar{\chi}_{1}\left(\partial_{t} \chi_{1}\right)-2^{\frac{1}{4}} i \sqrt{m} \bar{\xi}_{1}\left(\partial_{1}-i \partial_{2}\right) \chi_{1}-2^{\frac{1}{4}} i \sqrt{m} \bar{\chi}_{1}\left(\partial_{1}+i \partial_{2}\right) \xi_{1} \\
& \left.-2 \sqrt{2} m \bar{\xi}_{2} \xi_{2}+4 i m \bar{\chi}_{2}\left(\partial_{t} \chi_{2}\right)+2^{\frac{1}{4}} \sqrt{2 m} \bar{\xi}_{2}\left(\partial_{1}-i \partial_{2}\right) \chi_{2}-2^{\frac{1}{4}} \sqrt{2 m} \bar{\chi}_{2}\left(\partial_{1}+i \partial_{2}\right) \xi_{2}\right]
\end{aligned}
$$

Integrating out the non-dynamical fields $F_{1,2}$ and $\xi_{1,2}$ we find

$$
\begin{aligned}
S_{\text {kin }}= & \int d^{3} x\left[2 i m \bar{\varphi}_{1} \partial_{t} \varphi_{1}+\bar{\varphi}_{1} \partial_{i}^{2} \varphi_{1}-4 i m \bar{\varphi}_{2} \partial_{t} \varphi_{2}+\bar{\varphi}_{2} \partial_{i}^{2} \varphi_{2}\right. \\
& \left.+2 i m \bar{\chi}_{1} \partial_{t} \chi_{1}+\bar{\chi}_{1} \partial_{i}^{2} \chi_{1}+4 i m \bar{\chi}_{2} \partial_{t} \chi_{2}-\bar{\chi}_{2} \partial_{i}^{2} \chi_{2}\right]
\end{aligned}
$$


The component form of the interacting part of the action can be similarly obtained by means of the standard superspace manipulations combined with the Berezin integration (2.18). The result in terms of dynamical fields is

$$
\begin{aligned}
S_{\mathrm{int}}= & \int d^{3} x\left[-4|g|^{2}\left|\varphi_{1} \varphi_{2}\right|^{2}-|g|^{2}\left|\varphi_{1}\right|^{4}\right. \\
& -i g\left(\sqrt{2} \varphi_{1} \chi_{1}\left(\partial_{1}-i \partial_{2}\right) \bar{\chi}_{2}-2 \bar{\varphi}_{2} \chi_{1}\left(\partial_{1}-i \partial_{2}\right) \chi_{1}+2 \sqrt{2} \varphi_{1}\left(\left(\partial_{1}-i \partial_{2}\right) \chi_{1}\right) \bar{\chi}_{2}\right)+\text { h.c. } \\
& \left.+2|g|^{2}\left(-\left|\varphi_{1}\right|^{2} \bar{\chi}_{1} \chi_{1}-4\left|\varphi_{1}\right|^{2} \bar{\chi}_{2} \chi_{2}+2\left|\varphi_{2}\right|^{2} \bar{\chi}_{1} \chi_{1}+2 \sqrt{2} \varphi_{1} \varphi_{2} \bar{\chi}_{1} \bar{\chi}_{2}+2 \sqrt{2} \bar{\varphi}_{1} \bar{\varphi}_{2} \chi_{2} \chi_{1}\right)\right]
\end{aligned}
$$

\section{Quantum corrections in components}

As a cross-check of the results obtained in section 4 using superspace formalism, in this appendix we compute one and two-loop quantum corrections to the self-energies and oneloop corrections to the vertices using the component formalism.

We start from the action in components (B.5). Scalars and fermions share the same kinetic operator. Therefore, the tree-level propagators are

$$
\begin{aligned}
& \left\langle\varphi_{1}(\omega, \vec{p}) \bar{\varphi}_{1}(-\omega,-\vec{p})\right\rangle=\left\langle\chi_{1}(\omega, \vec{p}) \bar{\chi}_{1}(-\omega,-\vec{p})\right\rangle=\frac{i}{2 m \omega-\vec{p}^{2}+i \varepsilon} \\
& \left\langle\varphi_{2}(\omega, \vec{p}) \bar{\varphi}_{2}(-\omega,-\vec{p})\right\rangle=\left\langle\chi_{2}(\omega, \vec{p}) \bar{\chi}_{2}(-\omega,-\vec{p})\right\rangle=\frac{i}{4 m \omega-\vec{p}^{2}+i \varepsilon}
\end{aligned}
$$

Interaction vertices can be read directly from the lagrangian and are shown in figure 14 and 15, where we use dashed and continous lines to denote scalars and fermions, respectively.

In order to classify the admitted diagrams, we can take into account that the reduction in components does not affect the propagators as functions of $\omega$ and $\vec{p}$. Therefore, the arguments that led to formulate the fundamental selection rule 5.1 are still true. Moreover, the conservation of particle number at each vertex still provides the driving rule to select the admissible topologies and arrows configurations.

In order to properly define physical quantities and Green functions, we introduce renormalized fields and couplings defined as

$$
\left\{\begin{array}{l}
\varphi_{a}=Z_{a}^{-1 / 2} \varphi_{a}^{(B)}=\left(1-\frac{1}{2} \delta_{\varphi_{a}}\right) \varphi_{a}^{(B)} \quad a=1,2 \\
\chi_{a}=Z_{a}^{-1 / 2} \chi_{a}^{(B)}=\left(1-\frac{1}{2} \delta_{\chi_{a}}\right) \chi_{a}^{(B)} \\
m=Z_{m}^{-1} m^{(B)}=\left(1-\delta_{m}\right) m^{(B)} \\
g=\mu^{-\varepsilon} Z_{g}^{-1} g^{(B)}=\mu^{-\varepsilon}\left(1-\delta_{g}\right) g^{(B)}
\end{array}\right.
$$

Spatial integrals are computed in dimension $d=2-\varepsilon$ and we have introduced the mass scale $\mu$ to keep the coupling constant dimensionless.

One-loop corrections to the self-energies. By applying selection rule 5.1 and particle number conservation there are no admissible one-loop self-energy diagrams for particles in 

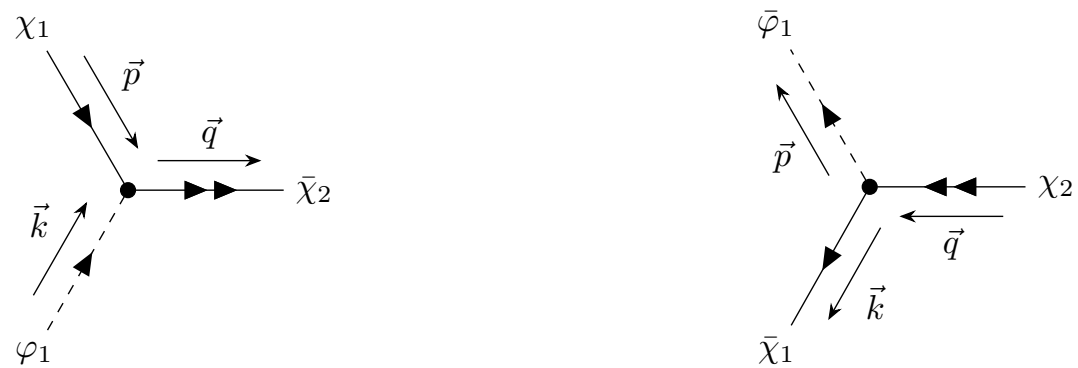

(a) $-\sqrt{2} i g\left[\left(q_{1}-i q_{2}\right)-2\left(p_{1}-i p_{2}\right)\right]$

(b) $\sqrt{2} i g^{*}\left[\left(q_{1}+i q_{2}\right)-2\left(k_{1}+i k_{2}\right)\right]$
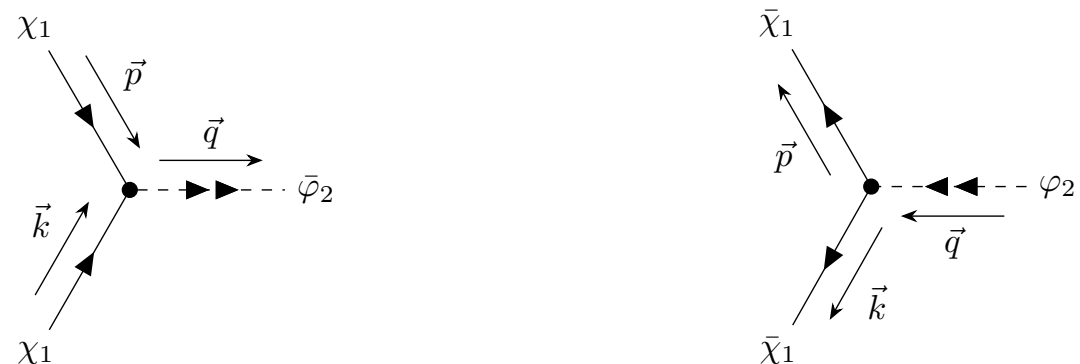

(c) $-2 i g\left[\left(k_{1}-i k_{2}\right)-\left(p_{1}-i p_{2}\right)\right]$

(d) $2 i g^{*}\left[\left(k_{1}+i k_{2}\right)-\left(p_{1}+i p_{2}\right)\right]$

Figure 14. Feynman rules for three-point vertices. Scalars are denoted by dashed lines, while fermions by continuous lines.

sector 1. For fields in sector 2, instead we find a non-vanishing contribution both for the scalar and the fermion corresponding to the diagrams in figure 16. Direct inspection leads to

$$
i \mathcal{M}_{\mathrm{b}}^{(2)}=i \mathcal{M}_{\mathrm{f}}^{(2)}=\frac{2|g|^{2}}{(2 \pi)^{3}} \int d \omega d^{2} k \frac{(\vec{p}-2 \vec{k})^{2}}{\left[2 m \omega-\vec{k}^{2}+i \varepsilon\right]\left[2 m(\Omega-\omega)-(\vec{p}-\vec{k})^{2}+i \varepsilon\right]}
$$

We use the residue method to perform the integration in $\omega$, obtaining

$$
\mathcal{M}^{(2)}=-\frac{|g|^{2}}{m} \int \frac{d^{2} k}{(2 \pi)^{2}} \frac{(\vec{p}-2 \vec{k})^{2}}{2 m \Omega-\vec{k}^{2}-(\vec{p}-\vec{k})^{2}+i \varepsilon}
$$

The remaining integral is UV divergent and can be computed with standard techniques of dimensional regularization. We obtain

$$
\mathcal{M}^{(2)}=\frac{|g|^{2}}{m} d \frac{\mu^{2(2-d)}}{(4 \pi)^{d / 2}} \Gamma\left(-\frac{d}{2}\right)\left(\frac{\vec{p}^{2}}{4}-m \Omega\right)^{\frac{d}{2}}=\frac{|g|^{2}}{2 \pi m}\left(2 m \Omega-\frac{\vec{p}^{2}}{2}\right) \frac{1}{\varepsilon}+\text { finite }
$$

In minimal subtraction scheme the $1 / \epsilon$ pole is cancelled by setting in (C.2)

$$
\delta_{\varphi_{2}}^{(1 \text { loop })}=\delta_{\chi_{2}}^{(1 \text { loop })}=-\frac{|g|^{2}}{4 \pi m} \frac{1}{\varepsilon}, \quad \delta_{m}^{(1 \text { loop })}=0
$$

whereas $\delta_{\varphi_{1}}^{(1 \text { loop })}=\delta_{\chi_{1}}^{(1 \text { loop })}=0$. This result is consistent with the one-loop renormalization (5.23) that we have found in superspace. 


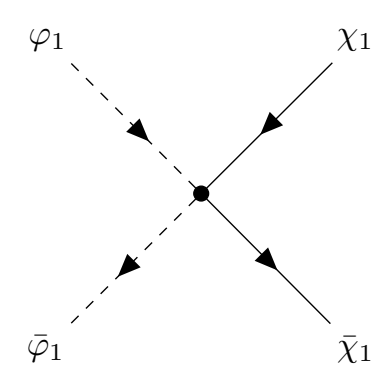

(a) $-2 i|g|^{2}$

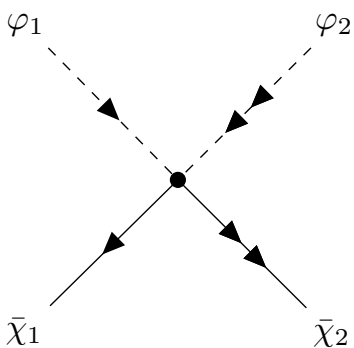

(d) $4 \sqrt{2} i|g|^{2}$

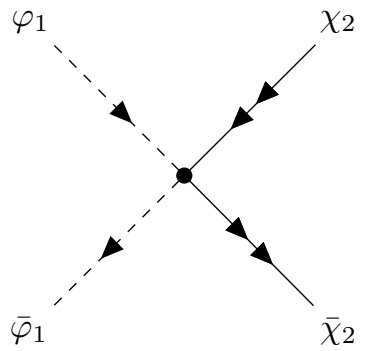

(b) $-8 i|g|^{2}$

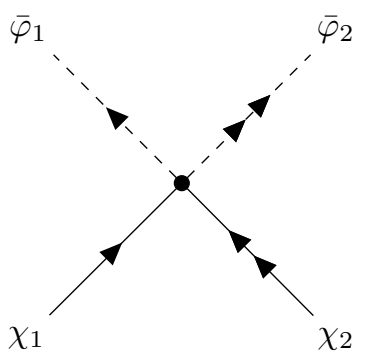

(e) $4 \sqrt{2} i|g|^{2}$

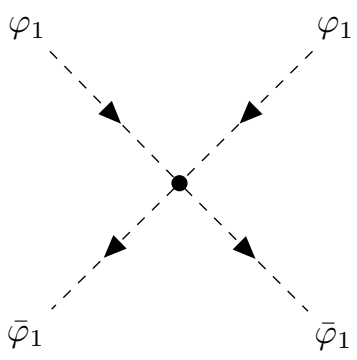

(g) $-4 i|g|^{2}$

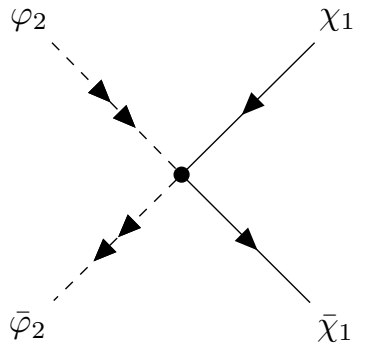

(c) $4 i|g|^{2}$

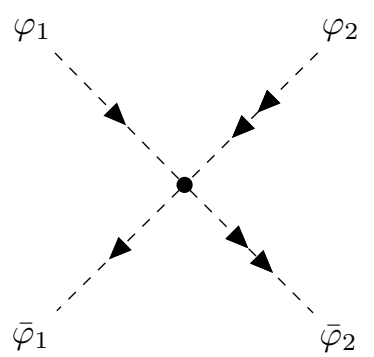

(f) $-4 i|g|^{2}$

Figure 15. Feynman rules for four-point vertices. Scalars are denoted by dashed lines, while fermions by continuous lines.

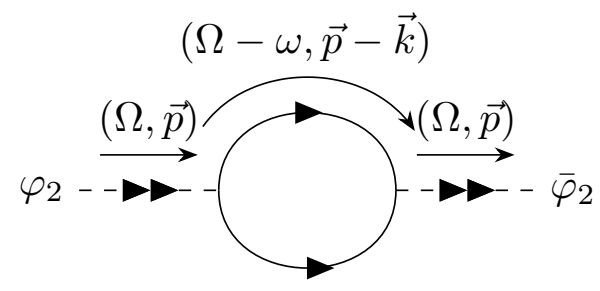

(a) $i \mathcal{M}_{\mathrm{b}}^{(2)}\left(\varphi_{2}, \bar{\varphi}_{2}\right)$

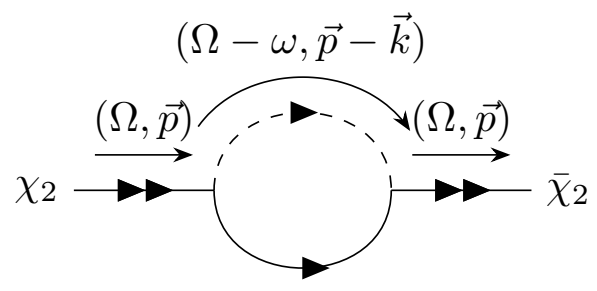

(b) $i \mathcal{M}_{\mathrm{f}}^{(2)}\left(\chi_{2}, \bar{\chi}_{2}\right)$

Figure 16. 1-loop correction to the scalar (a) and fermionic (b) self-energies in sector 2.

One-loop corrections to three-point vertices. In the action in components there are two types of three-point vertices (see figure 15). We discuss them separately.

Vertex $\mathbf{V}_{3}\left(\chi_{1}, \chi_{1}, \bar{\varphi}_{2}\right)$ and its complex conjugate are not corrected at one loop since we cannot build any diagram consistent with particle number conservation. It then follows 


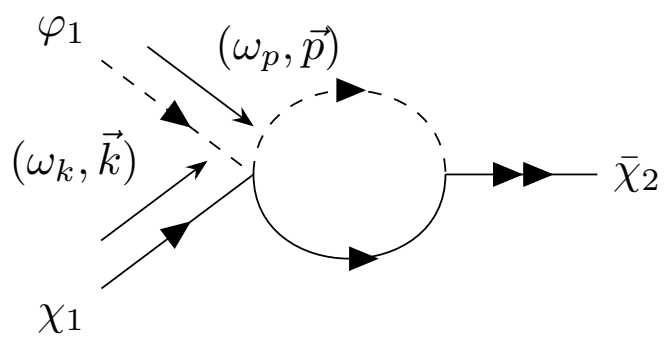

Figure 17. 1-loop correction to the 3-point vertex.

that

$$
\left.\left(\delta_{g}+\delta_{\chi_{1}}+\frac{1}{2} \delta_{\varphi_{2}}^{*}\right)\right|_{(1 \mathrm{loop})}=0
$$

Using result (C.6), we then find

$$
\delta_{g}^{(1 \text { loop })}=\frac{|g|^{2}}{8 \pi m} \frac{1}{\varepsilon}
$$

Vertex $\mathbf{V}_{3}\left(\varphi_{1}, \chi_{1}, \bar{\chi}_{2}\right)$ has in principle a one-loop contribution shown in figure 17 .

After $\omega$-integration by residues, this diagram gives

$$
\mathcal{M}^{(3)}\left(\varphi_{1}, \chi_{1}, \bar{\chi}_{2}\right)=-\frac{|g|^{2}}{m} \frac{\sqrt{2} g}{(2 \pi)^{2}} \int d^{2} l \frac{\left(p_{1}+k_{1}\right)-i\left(p_{2}+k_{2}\right)-2\left(l_{1}-i l_{2}\right)}{2 m\left(\omega_{p}+\omega_{k}\right)-\vec{l}^{2}-(\vec{p}+\vec{k}-\vec{l})^{2}+i \varepsilon}
$$

We perform dimensional regularization along the spatial directions. Since the integrand contains in the numerator an expression which depends explicitly on the $l_{i}$ components in a non-covariant way, before continuing the integrand to $d$ dimensions we need to give a prescription to covariantize the numerator. We introduce the vector $\vec{v}=(1,-i)$ and write the numerator as $\vec{v} \cdot(\vec{p}+\vec{k}-2 \vec{l})$. We then continue the integrand to $d$ dimensions, promoting also $\vec{v}$ to a $d$-dimensional vector with only the first two components different from zero. We have

$$
\mathcal{M}^{(3)}\left(\varphi_{1}, \chi_{1}, \bar{\chi}_{2}\right)=-\frac{|g|^{2}}{m} \frac{\sqrt{2} g \mu^{3(2-d)}}{(2 \pi)^{d}} \int d^{d} l \frac{\vec{v} \cdot(\vec{p}+\vec{k}-2 \vec{l})}{2 m\left(\omega_{p}+\omega_{k}\right)-\overrightarrow{l^{2}}-(\vec{p}+\vec{k}-\vec{l})^{2}+i \varepsilon}
$$

With the change of variables $\vec{q}=\vec{l}-\frac{\vec{p}+\vec{k}}{2}$ we obtain

$$
\mathcal{M}^{(3)}\left(\varphi_{1}, \chi_{1}, \bar{\chi}_{2}\right)=-\frac{|g|^{2}}{m} \frac{\sqrt{2} g \mu^{3(2-d)}}{(2 \pi)^{d}} \int d^{d} q \frac{\vec{v} \cdot \vec{q}}{q^{2}-m\left(\omega_{p}+\omega_{k}\right)+\frac{(\vec{p}+\vec{k})^{2}}{4}+i \varepsilon}
$$

This integral vanishes for symmetry reasons. The lack of one-loop corrections then implies that conterterms in eq. (C.2) need to satisfy

$$
\left.\left(\delta_{g}+\frac{1}{2} \delta_{\varphi_{1}}+\frac{1}{2} \delta_{\chi_{1}}+\frac{1}{2} \delta_{\chi_{2}}\right)\right|_{(1 \mathrm{loop})}=0
$$

This condition is automatically satisfied by results in eqs. (C.6), (C.8).

We note that the one-loop result $\delta_{g}=-\frac{1}{2} \delta_{\chi_{2}}$ is the component version of the superspace constraint (5.24). As expected, quantum corrections do not break supersymmetry. 

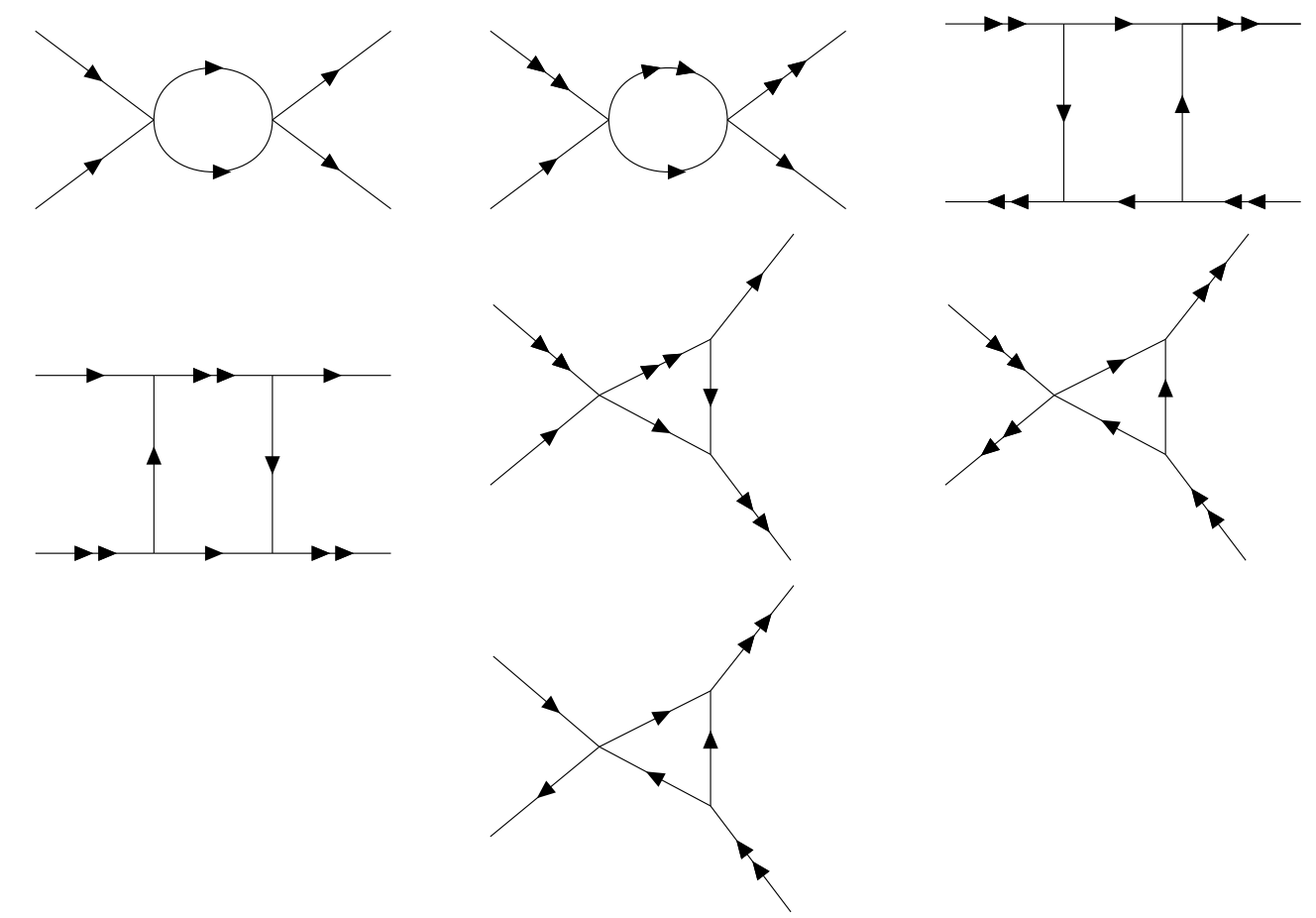

Figure 18. Possible topologies of one-loop corrections to four-point vertices for the dynamical fields. In the picture we do not distinguish between bosonic and fermionic lines.

One-loop corrections to four-point vertices. In principle, the one-loop evaluation of self-energies and three-point vertices allows to solve for all the unknowns in lagrangian (B.5). Moreover, we have verified that the corrections are all consistent between themselves and with the superspace results. However, we may want to consider some 1PI diagrams involving four-point vertices, in order to provide further evidence that SUSY is preserved also working in components.

Compared to the previous cases, we have far more possibilities to build four-point diagrams with the vertices at our disposal (see figures 14, 15). All the topologies of diagrams consistent with particle number conservation at each vertex are reported in figure 18.

For example, we consider the first diagram in figure 18, i.e. the one-loop correction to the vertex $\mathbf{V}_{4}\left(\varphi_{1}, \varphi_{1}, \bar{\varphi}_{1}, \bar{\varphi}_{1}\right)$. This is the only diagram among the many containing as external lines only fields from sector 1 . We report the precise assignments of momenta and energy in figure 19.

The $t$ and $u$-channel diagrams vanish because we have circulating arrows in the internal loop. After integration in $\omega$, the integral corresponding to the $s$-channel diagram is

$$
\mathcal{M}^{(4)}\left(\varphi_{1}, \varphi_{1}, \bar{\varphi}_{1}, \bar{\varphi}_{1}\right)=-\frac{4|g|^{4}}{m} \int \frac{d^{2} l}{(2 \pi)^{2}} \frac{1}{2 m\left(\omega_{p}+\omega_{k}\right)-\overrightarrow{l^{2}}-(\vec{p}+\vec{k}-\vec{l})^{2}+i \varepsilon}
$$

Performing the change of variables $\vec{q}=\vec{l}-\frac{\vec{p}+\vec{k}}{2}$, in dimensional regularization we can write

$$
\mathcal{M}^{(4)}\left(\varphi_{1}, \varphi_{1}, \bar{\varphi}_{1}, \bar{\varphi}_{1}\right)=\frac{4|g|^{4}}{m} \frac{\mu^{4(2-d)}}{(4 \pi)^{d / 2}} \frac{1}{\Gamma(d / 2)} \int_{0}^{\infty} d q \frac{q^{d-1}}{q^{2}-m\left(\omega_{p}+\omega_{k}\right)+\frac{(\vec{p}+\vec{k})^{2}}{4}+i \varepsilon}
$$




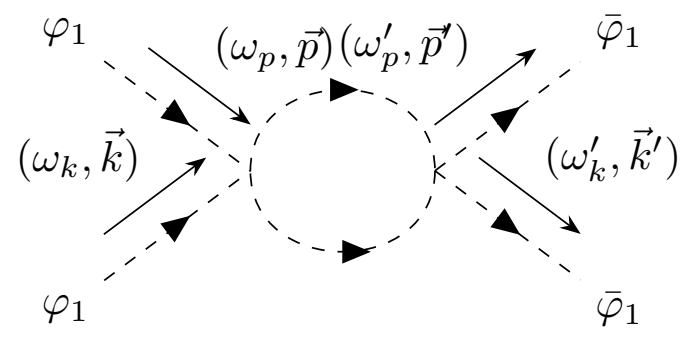

(a)

Figure 19. One-loop 1PI correction to the four-point vertex with external scalars from sector 1, coming from the $s$ channel.

After performing the last integration and expanding in $\varepsilon=2-d$ we find

$$
\mathcal{M}^{(4)}\left(\varphi_{1}, \varphi_{1}, \bar{\varphi}_{1}, \bar{\varphi}_{1}\right)=\frac{|g|^{4}}{\pi m} \frac{1}{\varepsilon}+\text { finite }
$$

The renormalization condition in minimal subtraction scheme requires

$$
\mathcal{M}^{(4)}\left(\varphi_{1}, \varphi_{1}, \bar{\varphi}_{1} \bar{\varphi}_{1}\right)-4|g|^{2}\left(2 \delta_{g}+2 \delta_{\varphi_{1}}\right)=0
$$

We then obtain

$$
\delta_{g}^{(1 \text { loop })}=\frac{|g|^{2}}{8 \pi m} \frac{1}{\varepsilon}
$$

which is consistent with (C.8).

This confirms that SUSY is preserved by quantum corrections. Moreover, the quantum corrections of the coupling constant $g$ are completely determined by the wave-function renormalization, as expected from the non-renormalization theorem.

Two-loop corrections to the self-energy. In component field formalism the number of Feynman diagrams at each loop order is much greater than using the superspace approach. This makes the evaluation of quantum corrections more involved when the number of loops increases. However, in the non-relativistic case selection rules 5.1 and 5.2 help in drastically decreasing the number of diagrams to be considered. In particular self-energies are easily treatable also at two loops. Here we report this calculation as an example of higher loop corrections in component field formalism.

At two loops the only self-energy diagram compatible with the selections rules is the one for the $\chi_{2}$ fermion, depicted in figure 20. Since there is no possibility to draw a nonvanishing diagram for the corresponding scalar, consistency with SUSY invariance requires this contribution to vanish. We now prove that this is indeed the case.

Writing down the corresponding integral and first performing the $\omega_{k}, \omega_{l}$ integrations by using the residue technique we find

$$
\mathcal{M}_{\mathrm{f}}^{(4)}\left(\chi_{2}, \bar{\chi}_{2}\right)=-\frac{|g|^{4}}{m^{2}} \int \frac{d^{2} k d^{2} l}{(2 \pi)^{4}} \frac{\vec{p}^{2}+4 \vec{l} \cdot \vec{k}+2(\vec{l}+\vec{k}) \cdot \vec{p}}{\left[2 m \Omega-\vec{k}^{2}-(\vec{p}-\vec{k})^{2}+i \varepsilon\right]\left[2 m \Omega-\vec{l}^{2}-(\vec{p}-\vec{l})^{2}+i \varepsilon\right]}
$$




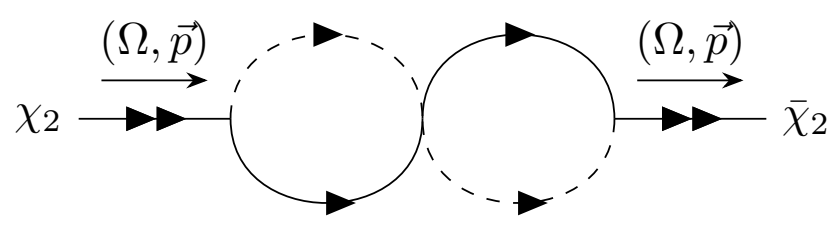

Figure 20. Two-loop correction to the self-energy for the dynamical fermion in sector 2 .
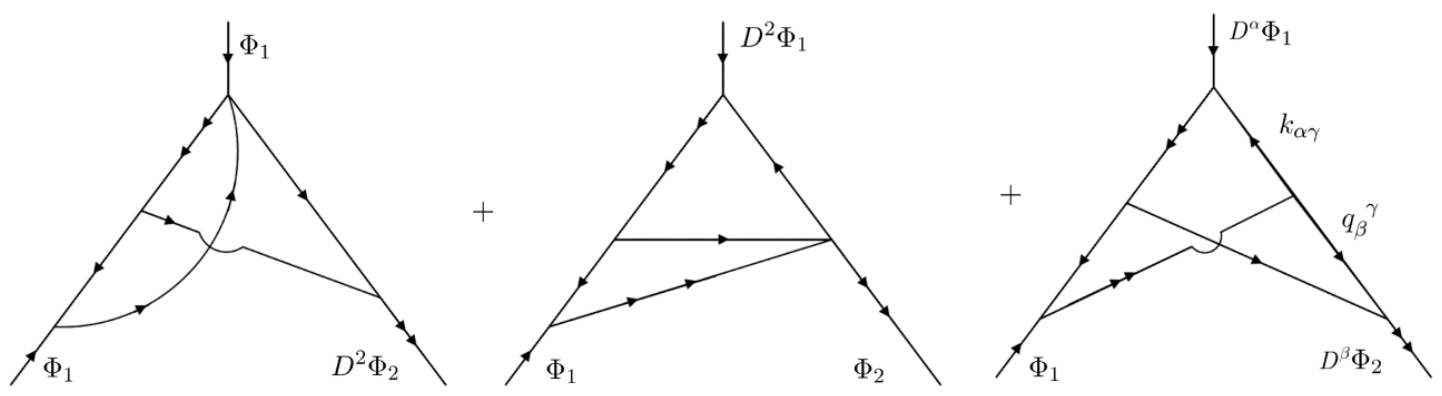

Figure 21. Diagrams resulting from the D-algebra reduction of diagram $13(\mathrm{~d})$.

Performing the change of variables

$$
\vec{k}=\vec{K}+\frac{\vec{p}}{2}, \quad \vec{l}=\vec{L}+\frac{\vec{p}}{2}
$$

and continuing the integral to $d=2-\epsilon$ dimensions we find

$$
\mathcal{M}_{\mathrm{f}}^{(4)}\left(\chi_{2}, \bar{\chi}_{2}\right)=-\frac{|g|^{4}}{m^{2}} \frac{\mu^{4(2-d)}}{(2 \pi)^{2 d}} \int d^{d} K d^{d} L \frac{4 \vec{K} \cdot \vec{L}}{\left[2 m \Omega-2 K^{2}-\frac{\vec{p}^{2}}{4}+i \varepsilon\right]\left[2 m \Omega-2 L^{2}-\frac{\vec{p}^{2}}{2}+i \varepsilon\right]}
$$

The two integrals vanish for symmetry reasons.

\section{Example of non-relativistic supergraph calculation}

As an example of supergraph calculation we consider the two-loop corrrection to the threepoint vertex.

Conservation of the particle number at each vertex restricts the allowed diagrams to the single non-planar graph of figure 13, where we have depicted all possible consistent assignments of arrows.

This is a case in which the number of chiral and anti-chiral vertices is different. Consequently, the factors of covariant derivatives are not only used to simplify propagators, but as the result of applying D-algebra (5.4), they give powers of momenta at the numerator which might affect the convergence of the $\omega$ integrations. Therefore, though all the diagrams contain closed loops of arrows and they should vanish due to selection rule 5.1, here we perform the explicit check.

For instance, focusing on the arrow configuration 13(d), the result of D-algebra is given in figure 21. 
In the first two diagrams the covariant derivatives act on the external fields or they are responsible for the simplification of some propagators. In fact, there are some effective 4 -point vertices due to Dirac $\delta$-functions arising in this way. In both cases we are left with a loop containing three propagators whose arrows form a closed loop, and then there is enough regularity to apply the Jordan's lemma and conclude that they vanish.

Due to the structure of the external covariant derivatives, the only relevant contribution from the third diagram in figure 21 is proportional to

$$
\begin{aligned}
& \int \frac{d \omega_{q} d^{2} q}{(2 \pi)^{3}} \frac{d \omega_{k} d^{2} k}{(2 \pi)^{3}} \epsilon_{\alpha \beta}\left(m\left(\omega_{k}+\omega_{q}\right)+\vec{k} \cdot \vec{q}\right) \frac{1}{2 m \omega_{k}-\vec{k}^{2}+i \varepsilon} \frac{1}{4 m\left(\omega_{p_{1}}+\omega_{k}\right)-\left(\overrightarrow{p_{1}}+\vec{k}\right)^{2}+i \varepsilon} \\
& \quad \times \frac{1}{2 m\left(\omega_{k}+\omega_{q}-\omega_{p_{2}}\right)-\left(\vec{k}+\vec{q}-\vec{p}_{2}\right)^{2}+i \varepsilon} \frac{1}{4 m\left(\omega_{k}+\omega_{q}\right)-(\vec{k}+\vec{q})^{2}+i \varepsilon} \\
& \quad \times \frac{1}{2 m \omega_{q}-\vec{q}^{2}+i \varepsilon} \frac{1}{2 m\left(\omega_{p_{1}}+\omega_{p_{2}}-\omega_{q}\right)-\left(\vec{p}_{1}+\vec{p}_{2}-\vec{q}\right)^{2}+i \varepsilon}
\end{aligned}
$$

where momenta $\left(\omega_{p_{a}}, \vec{p}_{a}\right), a=1,2$ refer to the external $\Phi_{1}, \Phi_{2}$ particles. At the numerator we have used the null reduction of the $4 \mathrm{~d}$ expression $k_{\alpha \dot{\alpha}} q_{\beta}^{\dot{\alpha}}=\left(\sigma^{M}\right)_{\alpha \dot{\alpha}}\left(\sigma^{N}\right)_{\beta}{ }^{\dot{\alpha}} k_{M} q_{N}$.

If we now focus on the $\omega_{k}$ integration, we see that in the region of large $\omega_{k}$ the worst integrand goes as $1 / \omega_{k}^{3}$. This allows to apply Jordan's lemma and compute the integral by residue theorem. Since all the poles are on the same side of the complex plane the result is zero.

The same pattern occurs for the other configurations of arrows in figure 13(a)-(c). This provides a check of selection rule 5.1 in this particular case.

Open Access. This article is distributed under the terms of the Creative Commons Attribution License (CC-BY 4.0), which permits any use, distribution and reproduction in any medium, provided the original author(s) and source are credited.

\section{References}

[1] G.W. Semenoff, Condensed Matter Simulation of a Three-dimensional Anomaly, Phys. Rev. Lett. 53 (1984) 2449 [INSPIRE].

[2] D.P. DiVincenzo and E.J. Mele, Self-consistent effective-mass theory for intralayer screening in graphite intercalation compounds, Phys. Rev. B 29 (1985) 1685 [INSPIRE].

[3] A.H. Castro Neto, F. Guinea, N.M.R. Peres, K.S. Novoselov and A.K. Geim, The electronic properties of graphene, Rev. Mod. Phys. 81 (2009) 109 [arXiv:0709.1163].

[4] D. Friedan, Z. Qiu and S.H. Shenker, Superconformal Invariance in Two-Dimensions and the Tricritical Ising Model, Phys. Lett. B 151 (1985) 37.

[5] T. Grover, D.N. Sheng and A. Vishwanath, Emergent Space-Time Supersymmetry at the Boundary of a Topological Phase, Science 344 (2014) 280 [arXiv:1301.7449] [INSPIRE].

[6] Y. Yu and K. Yang, Simulating Wess-Zumino Supersymmetry Model in Optical Lattices, Phys. Rev. Lett. 105 (2010) 150605 [arXiv:1005.1399] [INSPIRE]. 
[7] L. Huijse, B. Bauer and E. Berg, Emergent Supersymmetry at the Ising-Berezinskii-Kosterlitz-Thouless Multicritical Point, Phys. Rev. Lett. 114 (2015) 090404 [arXiv: 1403.5565] [INSPIRE].

[8] S.-K. Jian, Y.-F. Jiang and H. Yao, Emergent Spacetime Supersymmetry in 3D Weyl Semimetals and 2D Dirac Semimetals, Phys. Rev. Lett. 114 (2015) 237001 [arXiv: 1407.4497] [INSPIRE].

[9] A. Rahmani, X. Zhu, M. Franz and I. Affleck, Emergent Supersymmetry from Strongly Interacting Majorana Zero Modes, Phys. Rev. Lett. 115 (2015) 166401 [Erratum ibid. 116 (2016) 109901] [arXiv:1504.05192] [INSPIRE].

[10] J. Yu, R. Roiban and C.-X. Liu, 2 +1D Emergent Supersymmetry at First-Order Quantum Phase Transition, arXiv: 1902.07407 [INSPIRE].

[11] S.-S. Lee, Emergence of supersymmetry at a critical point of a lattice model, Phys. Rev. B 76 (2007) 075103 [cond-mat/0611658] [INSPIRE].

[12] C.R. Hagen, Scale and conformal transformations in galilean-covariant field theory, Phys. Rev. D 5 (1972) 377 [INSPIRE].

[13] R. Jackiw and S.-Y. Pi, Classical and quantal nonrelativistic Chern-Simons theory, Phys. Rev. D 42 (1990) 3500 [Erratum ibid. D 48 (1993) 3929] [InSPIRE].

[14] T. Mehen, I.W. Stewart and M.B. Wise, Conformal invariance for nonrelativistic field theory, Phys. Lett. B 474 (2000) 145 [hep-th/9910025] [INSPIRE].

[15] D.B. Kaplan, M.J. Savage and M.B. Wise, A New expansion for nucleon-nucleon interactions, Phys. Lett. B 424 (1998) 390 [nucl-th/9801034] [INSPIRE].

[16] Y. Nishida and D.T. Son, Unitary Fermi gas, $\epsilon$-expansion and nonrelativistic conformal field theories, Lect. Notes Phys. 836 (2012) 233 [arXiv:1004.3597] [INSPIRE].

[17] M. Geracie, D.T. Son, C. Wu and S.-F. Wu, Spacetime Symmetries of the Quantum Hall Effect, Phys. Rev. D 91 (2015) 045030 [arXiv:1407.1252] [INSPIRE].

[18] D.T. Son, Toward an AdS/cold atoms correspondence: A Geometric realization of the Schrödinger symmetry, Phys. Rev. D 78 (2008) 046003 [arXiv: 0804.3972] [INSPIRE].

[19] K. Jensen and A. Karch, Revisiting non-relativistic limits, JHEP 04 (2015) 155 [arXiv: 1412.2738] [INSPIRE].

[20] C. Duval, G. Burdet, H.P. Kunzle and M. Perrin, Bargmann Structures and Newton-cartan Theory, Phys. Rev. D 31 (1985) 1841 [InSPIRE].

[21] R. Puzalowski, Galilean Supersymmetry, Acta Phys. Austriaca 50 (1978) 45 [InSPIRE].

[22] T.E. Clark and S.T. Love, Nonrelativistic supersymmetry, Nucl. Phys. B 231 (1984) 91 [INSPIRE].

[23] J.A. de Azcarraga and D. Ginestar, Nonrelativistic limit of supersymmetric theories, J. Math. Phys. 32 (1991) 3500 [INSPIRE].

[24] A. Meyer, Y. Oz and A. Raviv-Moshe, On Non-Relativistic Supersymmetry and its Spontaneous Breaking, JHEP 06 (2017) 128 [arXiv: 1703.04740] [INSPIRE].

[25] M. Leblanc, G. Lozano and H. Min, Extended superconformal Galilean symmetry in Chern-Simons matter systems, Annals Phys. 219 (1992) 328 [hep-th/9206039] [INSPIRE]. 
[26] O. Bergman and C.B. Thorn, SuperGalilei invariant field theories in $(2+1)$-dimensions, Phys. Rev. D 52 (1995) 5997 [hep-th/9507007] [INSPIRE].

[27] J. Beckers and V. Hussin, Dynamical Supersymmetries of the Harmonic Oscillator, Phys. Lett. A 118 (1986) 319 [INSPIRE].

[28] J.P. Gauntlett, J. Gomis and P.K. Townsend, Particle Actions as Wess-Zumino Terms for Space-time (Super)symmetry Groups, Phys. Lett. B 249 (1990) 255 [INSPIRE].

[29] C. Duval and P.A. Horvathy, On Schrödinger superalgebras, J. Math. Phys. 35 (1994) 2516 [hep-th/0508079] [INSPIRE].

[30] S. Chapman, Y. Oz and A. Raviv-Moshe, On Supersymmetric Lifshitz Field Theories, JHEP 10 (2015) 162 [arXiv: 1508.03338] [INSPIRE].

[31] S.Y. Yong and D.T. Son, Effective field theory for one-dimensional nonrelativistic particles with contact interaction, Phys. Rev. A 97 (2018) 043630 [arXiv:1711.10517] [INSPIRE].

[32] E. Bergshoeff, J. Rosseel and T. Zojer, Newton-Cartan (super)gravity as a non-relativistic limit, Class. Quant. Grav. 32 (2015) 205003 [arXiv: 1505.02095] [INSPIRE].

[33] O. Aharony, A. Hanany, K.A. Intriligator, N. Seiberg and M.J. Strassler, Aspects of $N=2$ supersymmetric gauge theories in three-dimensions, Nucl. Phys. B 499 (1997) 67 [hep-th/9703110] [INSPIRE].

[34] Y. Nakayama, Superfield Formulation for Non-Relativistic Chern-Simons-Matter Theory, Lett. Math. Phys. 89 (2009) 67 [arXiv:0902.2267] [InSPIRE].

[35] J. Wess and B. Zumino, Supergauge Transformations in Four-Dimensions, Nucl. Phys. B 70 (1974) 39 [INSPIRE].

[36] S.J. Gates, M.T. Grisaru, M. Roček and W. Siegel, Superspace Or One Thousand and One Lessons in Supersymmetry, Front. Phys. 58 (1983) 1 [hep-th/0108200] [INSPIRE].

[37] M.T. Grisaru, W. Siegel and M. Roček, Improved Methods for Supergraphs, Nucl. Phys. B 159 (1979) 429 [INSPIRE].

[38] N. Seiberg, Naturalness versus supersymmetric nonrenormalization theorems, Phys. Lett. B 318 (1993) 469 [hep-ph/9309335] [INSPIRE].

[39] S. Weinberg, The quantum theory of fields. Vol. 3: Supersymmetry, Cambridge University Press, Cambridge U.K. (2005).

[40] R. Auzzi, S. Baiguera and G. Nardelli, Trace anomaly for non-relativistic fermions, JHEP 08 (2017) 042 [arXiv: 1705. 02229] [INSPIRE].

[41] L.F. Abbott and M.T. Grisaru, The Three Loop $\beta$-function for the Wess-Zumino Model, Nucl. Phys. B 169 (1980) 415 [inSPIRE].

[42] A. Sen and M.K. Sundaresan, The Four Loop Beta Function for the Wess-Zumino Model, Phys. Lett. B 101 (1981) 61.

[43] O. Bergman, Nonrelativistic field theoretic scale anomaly, Phys. Rev. D 46 (1992) 5474 [INSPIRE].

[44] K. Jensen, Anomalies for Galilean fields, SciPost Phys. 5 (2018) 005 [arXiv:1412.7750] [INSPIRE].

[45] I. Arav, S. Chapman and Y. Oz, Non-Relativistic Scale Anomalies, JHEP 06 (2016) 158 [arXiv: 1601.06795] [INSPIRE]. 
[46] R. Auzzi, S. Baiguera and G. Nardelli, On Newton-Cartan trace anomalies, JHEP 02 (2016) 003 [Erratum ibid. 1602 (2016) 177] [arXiv: 1511.08150] [INSPIRE].

[47] R. Auzzi and G. Nardelli, Heat kernel for Newton-Cartan trace anomalies, JHEP 07 (2016) 047 [arXiv: 1605. 08684] [INSPIRE].

[48] S. Pal and B. Grinstein, Heat kernel and Weyl anomaly of Schrödinger invariant theory, Phys. Rev. D 96 (2017) 125001 [arXiv:1703.02987] [InSPIRE].

[49] S. Pal and B. Grinstein, Weyl Consistency Conditions in Non-Relativistic Quantum Field Theory, JHEP 12 (2016) 012 [arXiv: 1605. 02748] [INSPIRE].

[50] R. Auzzi, S. Baiguera, F. Filippini and G. Nardelli, On Newton-Cartan local renormalization group and anomalies, JHEP 11 (2016) 163 [arXiv:1610.00123] [INSPIRE].

[51] Y. Nakayama, S. Ryu, M. Sakaguchi and K. Yoshida, A Family of super Schrödinger invariant Chern-Simons matter systems, JHEP 01 (2009) 006 [arXiv:0811.2461] [INSPIRE].

[52] Y. Nakayama, M. Sakaguchi and K. Yoshida, Interacting SUSY-singlet matter in non-relativistic Chern-Simons theory, J. Phys. A 42 (2009) 195402 [arXiv:0812.1564] [INSPIRE].

[53] K.-M. Lee, S. Lee and S. Lee, Nonrelativistic Superconformal M2-Brane Theory, JHEP 09 (2009) 030 [arXiv:0902.3857] [INSPIRE].

[54] C. Lopez-Arcos, J. Murugan and H. Nastase, Nonrelativistic limit of the abelianized ABJM model and the AdS/CMT correspondence, JHEP 05 (2016) 165 [arXiv:1510.01662] [INSPIRE].

[55] N. Doroud, D. Tong and C. Turner, On Superconformal Anyons, JHEP 01 (2016) 138 [arXiv: 1511.01491] [INSPIRE].

[56] O. Bergman and G. Lozano, Aharonov-Bohm scattering, contact interactions and scale invariance, Annals Phys. 229 (1994) 416 [hep-th/9302116] [INSPIRE].

[57] N. Doroud, D. Tong and C. Turner, The Conformal Spectrum of Non-Abelian Anyons, SciPost Phys. 4 (2018) 022 [arXiv: 1611.05848] [INSPIRE].

[58] C. Turner, Bosonization in Non-Relativistic CFTs, arXiv:1712.07662 [INSPIRE].

[59] S.P. Martin, A Supersymmetry primer, Adv. Ser. Direct. High Energy Phys. 21 (2010) 1 [Adv. Ser. Direct. High Energy Phys. 18 (1998) 1] [hep-ph/9709356]. 